\title{
TANAMI: tracking active galactic nuclei with austral milliarcsecond interferometry
}

\section{First-epoch $8.4 \mathrm{GHz}$ images}

\author{
R. Ojha ${ }^{1,2}$, M. Kadler ${ }^{3,4,5,6}$, M. Böck ${ }^{3,4}$, R. Booth ${ }^{7}$, M. S. Dutka ${ }^{8}$, P. G. Edwards ${ }^{9}$, A. L. Fey ${ }^{1}$, L. Fuhrmann ${ }^{10}$, \\ R. A. Gaume ${ }^{1}$, H. Hase ${ }^{11}$, S. Horiuchi ${ }^{12}$, D. L. Jauncey ${ }^{9}$, K. J. Johnston ${ }^{1}$, U. Katz $^{4}$, M. Lister ${ }^{13}$, J. E. J. Lovell ${ }^{14}$, \\ C. Müller ${ }^{3,4}$, C. Plötz ${ }^{15}$, J. F. H. Quick ${ }^{7}$, E. Ros ${ }^{16,10}$, G. B. Taylor ${ }^{17}$, D. J. Thompson ${ }^{18}$, S. J. Tingay ${ }^{19}$, G. Tosti ${ }^{20,21}$, \\ A. K. Tzioumis ${ }^{9}$, J. Wilms ${ }^{3,4}$, and J. A. Zensus ${ }^{10}$ \\ (Affiliations can be found after the references)
}

Received 19 June 2009 / Accepted 12 May 2010

\begin{abstract}
Context. A number of theoretical models vie to explain the $\gamma$-ray emission from active galactic nuclei (AGN). This was a key discovery of EGRET. With its broader energy coverage, higher resolution, wider field of view and greater sensitivity, the Large Area Telescope (LAT) of the Fermi Gamma-ray Space Telescope is dramatically increasing our knowledge of AGN $\gamma$-ray emission. However, discriminating between competing theoretical models requires quasi-simultaneous observations across the electromagnetic spectrum. By resolving the powerful parsecscale relativistic outflows in extragalactic jets and thereby allowing us to measure critical physical properties, Very Long Baseline Interferometry observations are crucial to understanding the physics of extragalactic $\gamma$-ray objects.

Aims. We introduce the TANAMI program (Tracking Active Galactic Nuclei with Austral Milliarcsecond Interferometry) which is monitoring an initial sample of 43 extragalactic jets located south of -30 degrees declination at $8.4 \mathrm{GHz}$ and $22 \mathrm{GHz}$ since 2007 . All aspects of the program are discussed. First epoch results at $8.4 \mathrm{GHz}$ are presented along with physical parameters derived therefrom.

Methods. These observations were made during 2007/2008 using the telescopes of the Australian Long Baseline Array in conjunction with Hartebeesthoek in South Africa. These data were correlated at the Swinburne University correlator.

Results. We present first epoch images for 43 sources, some observed for the first time at milliarcsecond resolution. Parameters of these images as well as physical parameters derived from them are also presented and discussed. These and subsequent images from the TANAMI survey are available at http://pulsar.sternwarte.uni-erlangen.de/tanami/.

Conclusions. We obtain reliable, high dynamic range images of the southern hemisphere AGN. All the quasars and BL Lac objects in the sample have a single-sided radio morphology. Galaxies are either double-sided, single-sided or irregular. About $28 \%$ of the TANAMI sample has been detected by LAT during its first three months of operations. Initial analysis suggests that when galaxies are excluded, sources detected by LAT have larger opening angles than those not detected by LAT. Brightness temperatures of LAT detections and non-detections seem to have similar distributions. The redshift distributions of the TANAMI sample and sub-samples are similar to those seen for the bright $\gamma$-ray AGN seen by LAT and EGRET but none of the sources with a redshift above 1.8 have been detected by LAT.
\end{abstract}

Key words. galaxies: active - galaxies: jets - galaxies: nuclei - gamma rays: observations - quasars: general

\section{Introduction}

Blazars are a radio-loud, violently variable, high-luminosity, high-polarization subset of Active Galactic Nuclei (AGN) that show luminosity variations across the electromagnetic spectrum. They typically exhibit apparent superluminal motion along the innermost parsecs of their radio jets. Their observed behaviour suggests they are very compact objects with parsec-scale jets oriented close to our line of sight. Despite decades of observations and modeling of the powerful relativistic jets of AGN, many fundamental questions about them remain unanswered. Jet composition, formation, and collimation are not well understood. Neither are the mechanisms responsible for their propagation, radiation, and interaction with their ambient medium.

The discovery by the EGRET detector onboard the Compton Gamma Ray Observatory (CGRO) that blazars can be bright $\gamma$-ray emitters (Hartman et al. 1992) was a major breakthrough in the study of AGN. Study of the $\gamma$-ray blazar population suggested that there is a close connection between $\gamma$-ray and radio emission, spectral changes, outbursts and the ejection of parsec-scale jet components (Dondi \& Ghisellini 1995). Many, but not all, of the radio brightest blazars have been detected at $\gamma$-ray energies. On the other hand, a small number of extragalactic jets which do not belong to the blazar class have been shown to be bright $\gamma$-ray sources, as well (e.g., NGC 1275 and Cen A; Abdo et al. 2009b). Currently there are a number of models attempting to explain the observed $\gamma$-ray emission of blazars and other extragalactic jets (see Böttcher 2007, for a review). Simultaneous broadband spectral energy distribution (SED) measurements across the electromagnetic spectrum are required to discriminate between such models.

Very Long Baseline Interferometry (VLBI) monitoring of blazars is a crucial component of this multiwavelength suite of observations as it provides the only direct measure of relativistic 
motion in AGN jets allowing us to calculate intrinsic jet parameters such as jet speed, Doppler factor, opening and inclination angles. They also provide the possibility of identifying the location and extent of emission regions. Thus VLBI observations constrain numerical jet simulations and provide tests of the relativistic-beam model (e.g., Cohen et al. 2007) that are not possible with any other observational technique.

The successful launch of the Fermi Gamma-ray Space Telescope, formerly known as GLAST (Gamma-ray Large Area Space Telescope; Gehrels \& Michelson 1999), on June 11th, 2008 was a major milestone in the quest to understand the connection between the low- and high-energy sections of blazar SEDs. The Large Area Telescope (LAT; Atwood et al. 2009) instrument on Fermi has broader energy coverage $(20 \mathrm{MeV}-300 \mathrm{GeV})$, higher resolution, wider field of view (over $20 \%$ of the sky) and a sensitivity $\sim 30$ times greater than EGRET. The combination of its wide field of view with a scanning pattern of observation means LAT observes the entire sky every $\sim 3 \mathrm{~h}$ making LAT capable of monitoring the sky on timescales from hours to years. Its higher sensitivity at higher energies allows LAT to measure high energy cutoffs which elucidate acceleration mechanisms, radiation and magnetic fields at the source.

The Tracking Active Galactic Nuclei with Austral Milliarcsecond Interferometry (TANAMI) program is a parsec-scale radio monitoring program targeting extragalactic jets south of -30 degrees declination. It uses the telescopes of the Australian Long Baseline Array (LBA, e.g., Ojha et al. 2004b), and other telescopes in South Africa, Antarctica and Chile to monitor an initial sample of 43 sources at approximately 2 -month intervals. The observations are typically made at two frequencies, $8.4 \mathrm{GHz}$ and $22 \mathrm{GHz}$, in order to calculate the spectral indices for the core as well as bright jet components. TANAMI began observations in November 2007 before the launch of Fermi so a proper observation cadence for the targets could be determined. To maximize the usage of TANAMI data, images of sources are made available at http://pulsar. sternwarte.uni-erlangen.de/tanami/as soon as they are available. The current target list of TANAMI is maintained at http://pulsar. sternwarte. uni-erlangen .de/tanami/ sample/.

While the immediate driver for TANAMI was the imminent launch of the Fermi Gamma-ray Space Telescope, these dualfrequency observations of morphology, motion, and other temporal variations of the most poorly studied third of the AGN sky have a number of other applications. One particularly exciting prospect is the possibility of combining $\gamma$-ray and radio monitoring of parsec-scale jet kinematics (and production) with a sensitive neutrino telescope. The ANTARES neutrino telescope (see http://antares.in2p3.fr), which is now fully operational and the KM3NeT detector (which has a projected completion date of 2011, see http://www.km3net.org) are being designed to detect neutrino point sources ${ }^{1}$. Extragalactic jets are among the most promising candidates to be neutrino point sources (Waxman 2007). Combined TANAMI, Fermi and ANTARES data will be used to search for neutrino signals correlated with $\gamma$-ray flares and epochs of jet production. The results of this work will be the subject of future publications.

In this paper, we describe how we selected our initial source sample (Sect. 2) and explain our observation and data reduction procedures (Sect. 3). We then present the first epoch images of 43 target sources at $8.4 \mathrm{GHz}$ in Sect. 4 followed by

\footnotetext{
${ }^{1}$ See KM3NeT Conceptual Design Report and references therein at http: //www . km3net . org/CDR/CDR-KM3NeT . pdf.
}

brief notes on individual sources (Sect. 5). This is followed by a discussion of our results (Sect. 6) and we end with our conclusions (Sect. 7). Throughout the paper we use the cosmology $H_{0}=73 \mathrm{~km} \mathrm{~s}^{-1} \mathrm{Mpc}^{-1}, \Omega_{\mathrm{m}}=0.27, \Omega_{\Lambda}=0.73$ where the symbols have their traditional meanings.

\section{Definition of the sample}

The TANAMI sample has been defined as a hybrid radio and $\gamma$-ray selected sample of AGN south of $\delta=-30^{\circ}$. Its main components are I) a radio selected flux-density limited subsample and II) a $\gamma$-ray selected subsample of known and candidate $\gamma$-ray sources based on results of $C G R O / E G R E T$. The radio subsample includes all sources (within our declination range) from the catalogue of Stickel et al. (1994) above a limiting radio flux density of $S_{5 \mathrm{GHz}}>2 \mathrm{Jy}$, which have a flat radio spectrum $(\alpha>$ $-0.5, S \sim v^{+\alpha}$ ) between $2.7 \mathrm{GHz}$ and $5 \mathrm{GHz}$. The 21 sources selected according to this radio flux-density criterion represent a Southern-hemisphere extension of the MOJAVE 1 sample (Lister et al. 2009a), which is complete in the declination range $0^{\circ}>\delta>-20^{\circ}$ down to the same flux-density limit at $15 \mathrm{GHz}$. The $\gamma$-ray selected subsample includes all known $\gamma$-ray blazars detected by EGRET south of $\delta=-30^{\circ}$, both the high-confidence and low-confidence associations made by Hartman et al. (1999), Tornikoski et al. (2002), Sowards-Emmerd et al. (2004) and Bignall et al. (2008). In addition, we have also included 4 known intra-day variable (IDV) sources (0405-385, 1144-379, 1257-326, and 1323-526) and 8 other sources, which either share the radio properties of EGRET-detected blazars at lower radio flux density or which represent prototypical examples of other AGN classes such as the bright and nearby radio galaxy Pictor A (0518-458) or the gigahertz peaked spectrum (GPS) source NGC 6328 (1718-649). In total, this sample contains 44 objects $^{2}$. Many of the sources in this sample have been well studied with VLBI in the past (Tingay et al. 1996a; Shen et al. 1997, 1998b; Tingay et al. 2002; Ojha et al. 2004a, 2005; Scott et al. 2004; Horiuchi et al. 2004; Dodson et al. 2008) but for about $30 \%$ only very limited information at typically much lower resolutions and image fidelity are available in the literature.

Most AGN with bright compact radio emission are strongly variable. This led the MOJAVE team to define their statistically complete radio-selected sample based on radio light curve observations of a large sample of sources over a ten-year time baseline and to include all sources that exceeded their flux-density limit at any epoch during this period. In contrast to this, the TANAMI sample is not statistically complete. However, based on experience from the transition of the original VLBA $2 \mathrm{~cm}$ Survey sample into the MOJAVE sample (Lister et al. 2009a), we can consider the radio-selected subsample of TANAMI as being representative of a complete sample. In a similar sense, the Fermi LBAS (LAT bright AGN sample) list is not statistically complete (Abdo et al. 2009b) and neither is the subsample of LBASTANAMI sources. A few bright $\gamma$-ray sources have no or only low-confidence associations ${ }^{3}$ and in addition, the presence of

\footnotetext{
2 The candidate EGRET $\gamma$-ray blazar 0527-359 was not observed due to scheduling problems.

3 Abdo et al. (2009a) list three sources below declination - 30 degrees and at high galactic latitude $|b|>10$ degrees, which have no association whatsoever and might be unknown AGNs: 0FGL J 0614.3-3330, 0FGL J 1311.9-3419, and 0FGL J2241.7-5239. Two sources in this region do have only low-confidence associations with AGN (0FGL J0407.6-3829 associated with PKS 0405-385 and 0FGL J 0412.9-5341 associated with PMN J 0413-5332).
} 
Table 1. The long baseline array and affiliated telescopes.

\begin{tabular}{lrl}
\hline \hline Telescope & $\begin{array}{r}\text { Diameter } \\
\text { (meters) }\end{array}$ & Location \\
\hline Parkes & 64 & Parkes, New South Wales, Australia \\
ATCA & $5 \times 22$ & Narrabri, New South Wales, Australia \\
Mopra & 22 & Coonabarabran, New South Wales, Australia \\
Hobart & 26 & Mt. Pleasant, Tasmania, Australia \\
Ceduna & 30 & Ceduna, South Australia, Australia \\
Hartebeesthoek $^{a}$ & 26 & Hartebeesthoek, South Africa \\
DSS43 & 70 & Tidbinbilla, ACT, Australia \\
DSS45 $^{b}$ & 34 & Tidbinbilla, ACT, Australia \\
O'Higgins $^{c}$ & 9 & O'Higgins, Antarctica \\
TIGO $^{c}$ & 6 & Concepcion, Chile \\
\hline
\end{tabular}

Notes. ${ }^{(a)}$ Not available since September 2008. ${ }^{(b)}$ Operated by the Deep Space Network of the National Aeronautics and Space Administration. (c) Operated by Bundesamt für Kartographie und Geodsie (BKG) http://www.bkg.bund.de/nn_147094/EN/Home/homepage__node. html__nnn=true.

known EGRET sources, which are not in the LBAS ${ }^{4}$, indicates the effect of long-term variability being also important in the $\gamma$-ray regime. We are working on improving the completeness of the $\gamma$-ray selected TANAMI subsample in collaboration with the Fermi/LAT science team. In addition to the 44 sources of the initial TANAMI sample, we have begun observations of 19 additional Fermi sources in November 2008, which will be reported elsewhere. Most of these sources have not been observed with VLBI before. We plan to continue adding new Fermi-detected sources to the TANAMI list through 2009.

The Veron-Veron 12th edition catalog (Veron-Cetty \& Veron 2006) was used to obtain optical classifications. The sample contains 24 quasars (optically unresolved broad-emission line objects), 6 BL Lac objects (optically unresolved sources with weak or absent emission lines), and 10 radio galaxies (optical galaxies, which are identified with radio sources). Three sources are unclassified.

After the beginning of Fermi science operations and the publication of the first list of bright $\gamma$-ray emitting AGN detected by Fermi/LAT between August and October 2008 (Abdo et al. 2009b), our sample-selection criteria have proved to be highly efficient in picking up bright $\gamma$-ray emitters. Our original sample contains 10 of the 18 high-confidence associations of the LBAS sample within our declination range, one out of two lower confidence association and one additional bright $\gamma$-ray source association (1759-396) outside the galactic latitude range considered by Abdo et al. (2009a). Four of these 12 sources have entered the TANAMI sample because they belong to both the radio flux-density limited sample and to the EGRET sample, two and four belong only to the radio or EGRET sample, respectively, and two belong to the IDV class. Details are given in Table 3. Notably, one out of only two LBAS radio galaxies belongs to the TANAMI sample (Cen A), as well as two out of seven highfrequency peaked BL Lac objects, which are also detected in the TeV energy range (2005-489 and 2155-304).

\footnotetext{
${ }^{4}$ Below declination -30 degrees, there are three 3EG sources (Hartman et al. 1999), which are not part of the LBAS sample: 0454-463, 1424-418, and 1933-400. nine other sources associated with lower confidence with EGRET sources by Tornikoski et al. (2002) and Sowards-Emmerd et al. (2004) have also not been seen as bright $\gamma$-ray sources by LAT during its first three months.
}

\section{Observations and data reduction}

TANAMI observations are made using the five telescopes of the Australian Long Baseline Array $\left(\mathrm{LBA}^{5}\right.$ ) along with other affiliated telescopes. Within Australia, TANAMI has periodic access to the $70 \mathrm{~m}$ and the $34 \mathrm{~m}$ telescopes at NASA's Deep Space Network (DSN) located at Tidbinbilla, near Canberra in the Australian Capital Territory (ACT). When available, these telescopes add crucial sensitivity to map details of the jet structure with higher fidelity, besides improving the $(u, v)$-coverage. Through September 2008, the highest resolution, intercontinental baselines were provided by the $26 \mathrm{~m}$ telescope in Hartbeesthoek, South Africa. However, this telescope experienced a major failure of a polar shaft bearing in October 2008 and is likely to remain unavailable for some time (Jonathan Quick, personal communication). Fortunately, through a successful International VLBI Service (IVS) proposal we have obtained access to two telescopes, O'Higgins, Antarctica and TIGO (Transportable Integrated Geodetic Observatory), Chile. Both these telescopes are operated by the Bundesamt für Kartographie und Geodäsie (BKG), the federal agency responsible for cartography and geodesy in Germany. These two telescopes greatly improve our $(u, v)$-coverage and partially offset the loss of Hartebeesthoek. Details of all of these telescopes used in TANAMI observations are summarized in Table 1. The telescopes participating in the observations reported in this paper are indicated in Table 2.

TANAMI observations are made at two frequencies: $8.4 \mathrm{GHz}$ ( $X$-band) and at $22 \mathrm{GHz}$ ( $K$-band). Imaging observations at $8.4 \mathrm{GHz}$ generally yield the best image fidelity and thus the most detailed structural information with this array as demonstrated, for example, by the US Naval Observatory/ATNF, International Celestial Reference Frame (ICRF) imaging program (Ojha et al. 2004a, 2005). This frequency is high enough to provide good resolution and low enough to avoid missing extended structure that typically has a steep spectrum. Atmospheric effects are also negligible at this frequency. Imaging observations at $22 \mathrm{GHz}$ are challenging but feasible (Tingay et al. 2003b). Not only do $22 \mathrm{GHz}$ images show morphology closer to the cores of the AGN, in combination with the $8 \mathrm{GHz}$ images, they yield spectral

\footnotetext{
5 The Long Baseline Array is part of the Australia Telescope which is funded by the Commonwealth of Australia for operation as a National Facility managed by CSIRO.
} 
Table 2. Summary of observations.

\begin{tabular}{lll}
\hline \hline Epoch & Participating Telescopes & Remarks \\
\hline 2007 Nov. 10 & Parkes, ATCA, Mopra, Hobart, Ceduna, Hartebeesthoek & $\ldots$ \\
2008 Feb. 07 & Parkes, ATCA, Mopra, Hobart, Ceduna, Hartebeesthoek, DSS43 & Hart for 15 and DSS43 for 5 h only \\
2008 Mar. 28 & Parkes, ATCA, Mopra, Hobart, Ceduna, Hartebeesthoek, DSS45 & DSS45 intermittent \\
2008 Jun. 09 & Parkes, ATCA, Mopra, Hobart, Ceduna, Hartebeesthoek & $\ldots$ \\
\hline
\end{tabular}

information on the core and bright jet components. These are a critical component of the broadband SEDs needed to understand the energetics of AGN. One 24-h epoch at each frequency is observed approximately every two months. In this paper, we describe the $8.4 \mathrm{GHz}$ observations. Table 2 summarizes the observations which are reported here.

For the array described above, the typical angular resolution (synthesized beam) achieved by TANAMI is $1.5-4$ by 0.5-1.0 mas in size, with the highest resolution in the east-west direction. Each source was observed in about 6 scans of approximately $10 \mathrm{~min}$ each. The data were recorded on the LBADRs (Long Baseline Array Disk Recorders) and correlated on the DiFX software correlator (Deller et al. 2007) at Swinburne University in Melbourne, Victoria. From November 2008 on, the data is being correlated at Curtin University in Perth, Western Australia.

The correlated data were loaded into AIPS using the locally written task ATLOD which is needed to read the data in the format that the LBA generates. Thereafter, data inspection, initial editing and fringe fitting was done in the standard manner using the National Radio Astronomy Observatory's Astronomical Image Processing System (AIPS) software (Greisen 1998). Observations of known sources with $\geq 90 \%$ of their correlated flux in a compact core were used to improve overall amplitude calibration. For each antenna, a single amplitude gain correction factor was derived based on fitting a simple Gaussian source model to the visibility data of the respective compact source after applying only the initial calibration based on the measured system temperatures and gain curves. Based on the differences between the observed and model visibilities, gain correction factors were calculated and the resulting set of amplitude gain correction factors was then applied to the visibility data of the target sources. The accuracy of the absolute amplitude calibration is conservatively estimated to be $20 \%$.

The imaging was performed applying standard methods using the program DIFMAP (Shepherd 1997). Specifically, the data were averaged into $30 \mathrm{~s}$ bins and then imaged using the CLEAN algorithm, giving the same weight to all visibility data points (natural weighting) and making use of phase self-calibration. The best model that could be obtained in this initial cycle of the hybrid mapping process was used to self calibrate the visibility amplitudes by applying time-independent gain factors for each antenna in the array. The model was then cleared and the resulting improved data were imaged in additional hybridmapping cycles following the same strategy but using timedependent gain factor functions with subsequently smaller time intervals $(180,60,20,5,2,1 \mathrm{~min})$. Before beginning a new cycle, the data were examined and edited if necessary. The images shown in Figs. 1 through 8 result from the final hybrid-mapping cycle using natural weighting and a 30 s solution interval. Some sources exhibit diffuse large-scale emission which could only be recovered by down-weighting the data on the longest baselines $((u, v)$-tapering). In Figs. 9-11 we show tapered images for 13 sources.

\section{Results}

Physical characteristics of the TANAMI sources, where available, are summarized in Table 3. The table lists their IAU source designation followed by their alternate name (where appropriate) and their Right Ascension and Declination in J2000.0 coordinates. Their optical identification, $V$-magnitude and redshift are listed in successive columns. The last four columns indicate which sources belong to the radio and $\gamma$-ray selected subsamples and whether they were detected by EGRET and LAT, respectively, where the LAT-detection flag refers to the bright-source list of Abdo et al. (2009b) based on the first three months of Fermi observations.

Figures 1 through 8 show contour plots of the 43 sources of the initial TANAMI sample. These images are made with natural weighting. A subset of the sources that have diffuse largescale emission are shown in Figs. 9-11 using $(u, v)$-tapering. The scale of each image is in milliarcseconds. The FWHM Gaussian restoring beam applied to the images is shown as a hatched ellipse in the lower left of each panel. Each panel also shows a bar representing a linear scale of $1 \mathrm{pc}, 10 \mathrm{pc}$, or $100 \mathrm{pc}$ depending on the source extent and distance, except for the sources without a measured redshift. The average root-mean-square (RMS) noise in the images is $\sim 0.43 \mathrm{mJy}^{\text {beam }}{ }^{-1}$ with a median RMS of $\sim 0.33 \mathrm{mJy}^{\text {beam }}{ }^{-1}$.

Image parameters are listed in Table 4 . The first two columns list the IAU source name and the epoch of the image shown. The lowest contour level is at 3 times the root-mean-square noise and is listed in Col.(3). The peak flux density in each image and the total flux are given in Col. (4) and Col. (5). The major axis, minor axis, and position angle of the restoring beam are in Cols. (6)-(8). Columns (9) and (10) show the structural classification and the core brightness temperature respectively. Both of these were evaluated using criteria described in Sect. 6. The final two columns describe the core luminosity and total luminosity of each source. Table 5 summarizes the image parameters for the 13 sources for which we present tapered images. The first eight columns are identical to those of Table 4. The last column indicates the baseline length in $M \lambda$ at which the visibility data were down weighted to $10 \%$.

Adequate $(u, v)$-coverage is a key consideration for any VLBI survey. It is a particular concern when observing with the LBA since the LBA is an ad hoc array and the locations of its constituent telescopes are not ideal for producing uniform $(u, v)$-coverage. Representative plots of the $(u, v)$-coverage for four sources with declinations spanning the range of the TANAMI sample are shown in Fig. 12. The shorter baselines at the center of each plot are those between telescopes within Australia. The long baselines at the periphery of each figure are those to the Hartebeesthoek telescope in South Africa. The absence of intermediate-length baselines between the shorter intra-Australian and the trans-oceanic baselines remains the most important limiting factor on image quality. However, as past imaging programs have found (e.g., Ojha et al. 2005), 

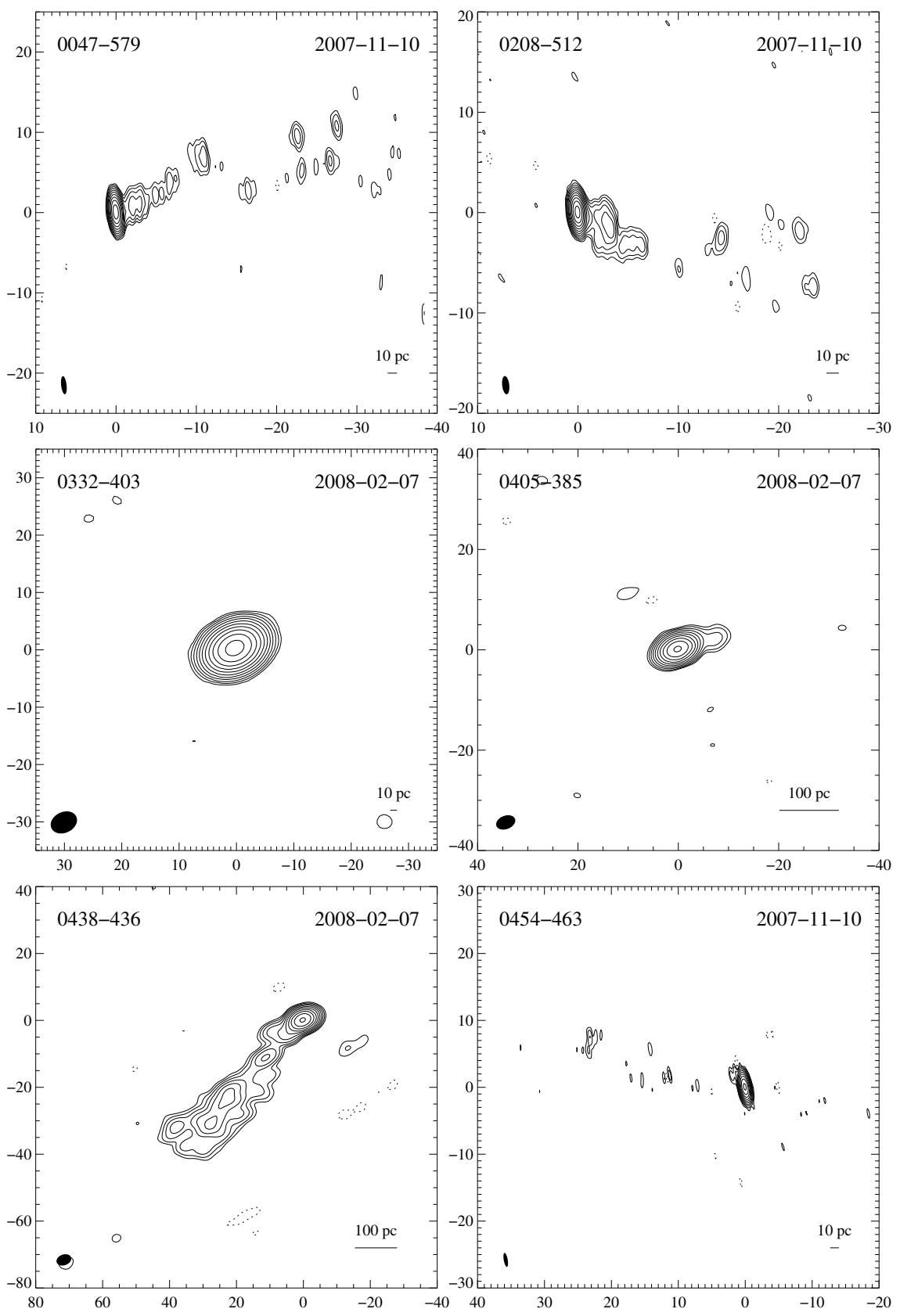

Fig. 1. Contour maps of the 43 TANAMI sources at $8.4 \mathrm{GHz}$. The scale of each image is in milliarcseconds. The $F W H M$ Gaussian restoring beam applied to the images is shown as a hatched ellipse in the lower left of each panel. Each panel also shows a bar representing a linear scale between $0.1 \mathrm{pc}$ and $100 \mathrm{pc}$ except for the sources without a measured redshift.

this constraint does not preclude good images provided special care is taken in both the calibration and imaging process. Each epoch of observation included two sources which are mutually visible to the LBA and VLBA. Our LBA images of these two sources were checked for consistency with nearcontemporaneous VLBA images and revealed no problems.

\section{Notes on individual sources}

In this section we describe the morphology of each source after a brief summary of its background and past observations where present.
0047-579 This source is a bright high-redshift $(z=1.8)$ quasar from the radio-selected subsample, which has not yet been seen by Fermi in its initial bright-source list (Abdo et al. 2009b). The 8.4 GHz VLBI image by Ojha et al. (2005) shows a compact core with a second component located $\sim 20$ mas to the west. Our TANAMI image (Fig. 1) at the same frequency is at higher resolution and shows a continuous well-collimated jet from the core toward the outer component seen by Ojha et al. (2005), which is partially resolved by the TANAMI array. The tapered image (Fig. 9) reveals more extended emission along the jet position angle out to $\sim 40$ mas from the core.

0208-512 This bright and highly polarized quasar (Impey \& Tapia 1988, 1990) was a known EGRET source 

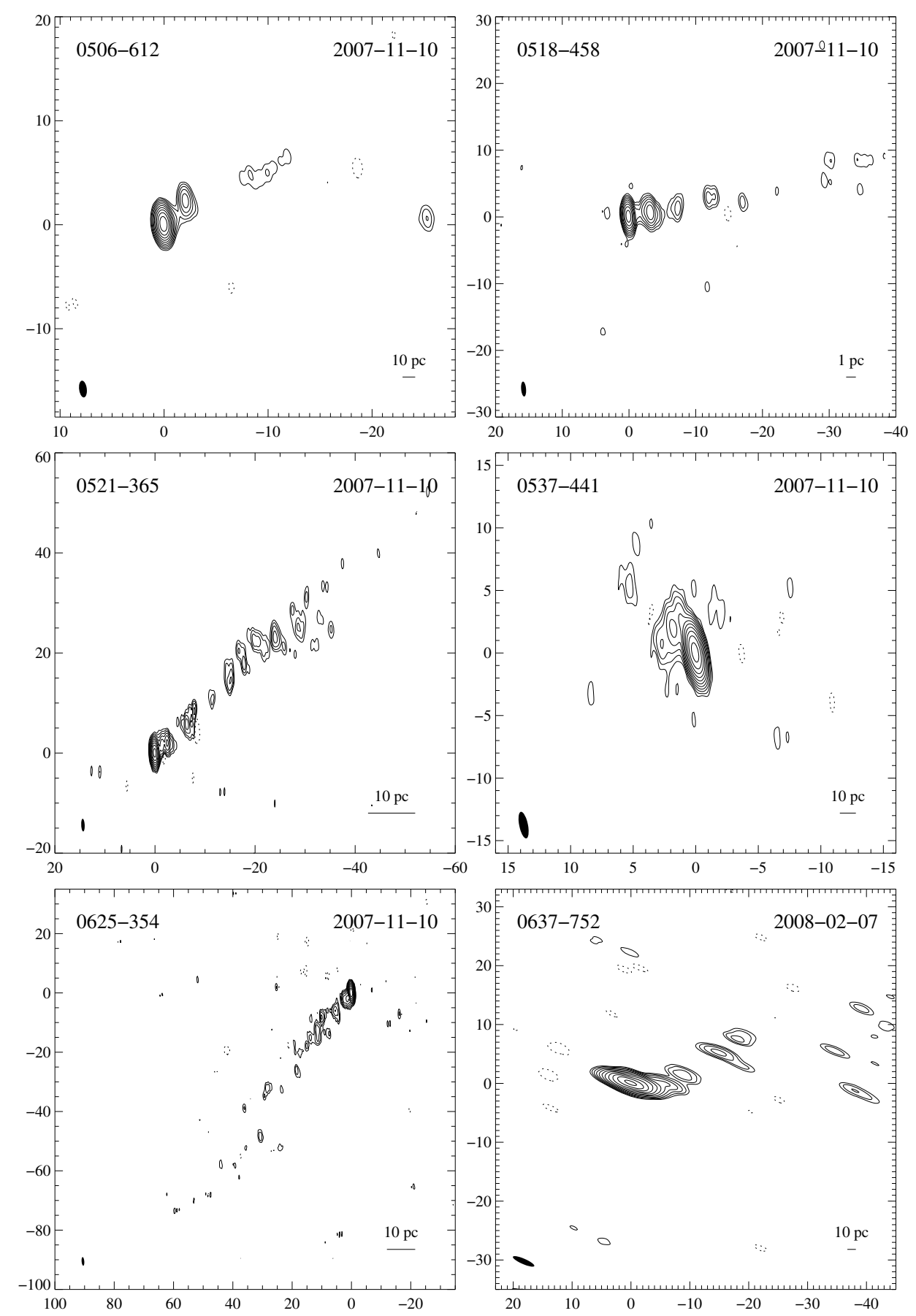

Fig. 2. Same as Fig. 1

(Hartman et al. 1999) with its $\gamma$-ray spectrum being one of the hardest detected AGN spectra in this energy range (von Montigny et al. 1995; Chiang et al. 1995). Right after the beginning of the Fermi mission, 0208-512 was detected as a flaring $\gamma$-ray source by the LAT (Tosti 2008) and it is also a member of the 3-month LAT bright source list sample (Abdo et al. 2009b). Shen et al. (1998a) find a lower limit for the Doppler factor of 10.2 using the ROSAT measurements $(0.22 \mu \mathrm{Jy}$ at $1 \mathrm{keV})$ of Dondi \& Ghisellini (1995). From model fit parameters of the 1992 and 1993 data they obtained a proper motion of $0.6 \pm 0.7 \mathrm{mas} / \mathrm{yr}$ corresponding to a transverse velocity of $16.8 \mathrm{c}(17 \pm 20 \mathrm{c})^{6}$. Tingay et al. (2002) find no significant

${ }^{6}$ For $H_{0}=100$ and $q_{0}=0.5$. detection of component proper motion in this source. Shen et al. (1998b) and Ojha et al. (2004a) find a slight extension of the compact core to the southwest. In the X-ray regime Marshall et al. (2005) find a 4 arcsec jet to the southwest in addition to the core emission. Our image (Fig. 1) is consistent but more sensitive showing a continuous well-collimated twisting jet extending southwest to about $7 \mathrm{~ms}$ with diffuse emission beyond that to over $20 \mathrm{~ms}$ from the core. Given the high fidelity of this image further TANAMI epochs should allow us to pin down its expected superluminal motion.

0332-403 This BLLac object has not been detected by EGRET but was detected with Fermi between August and 

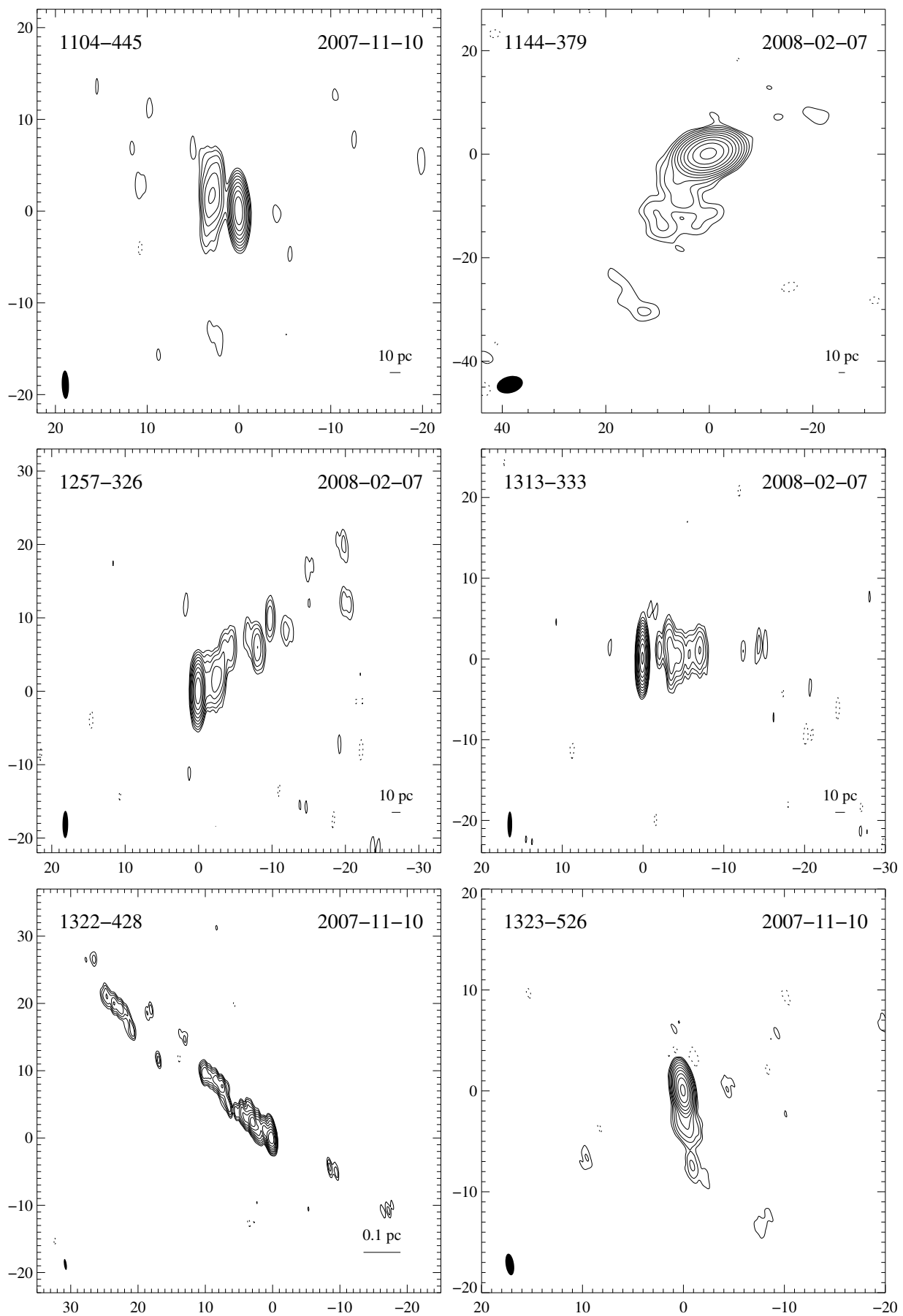

Fig. 3. Same as Fig. 1.

October 2008 (Abdo et al. 2009b). The source is very compact (Ojha et al. 2004a) with previous reports of weak and short extensions to the east (Shen et al. 1998b), and to the west (Fomalont et al. 2000), respectively. Our TANAMI image of this source has considerably lower resolution than most of our other images because no trans-oceanic baselines were available and does not resolve the source.

0405-385 This source is one of three unusually strong intraday variable (IDV) sources (Kedziora-Chudczer 2006) ${ }^{7}$. It was

\footnotetext{
7 The other two strong IDV sources are PKS 1257-326 and $\mathrm{J} 1819+385$.
}

not detected by EGRET but has been detected, with low confidence, by the LAT (Abdo et al. 2009b). This is a very compact source but Zensus et al. (2002) find a component 1.5 mas to the west of the core. Similarly, Ojha et al. (2004a) present a component 4 mas west of their core. For the first-epoch TANAMI image of this source, the absence of trans-oceanic baselines has resulted in lower resolution, yielding a compact structure with an extension to the west.

0438-436 The VLBI image of this high-redshift quasar by Preston et al. (1989) shows two components, the core and an additional component 35 mas to the southeast. A map with better resolution by Shen et al. (1998b) resolves the component 
A\&A 519, A45 (2010)
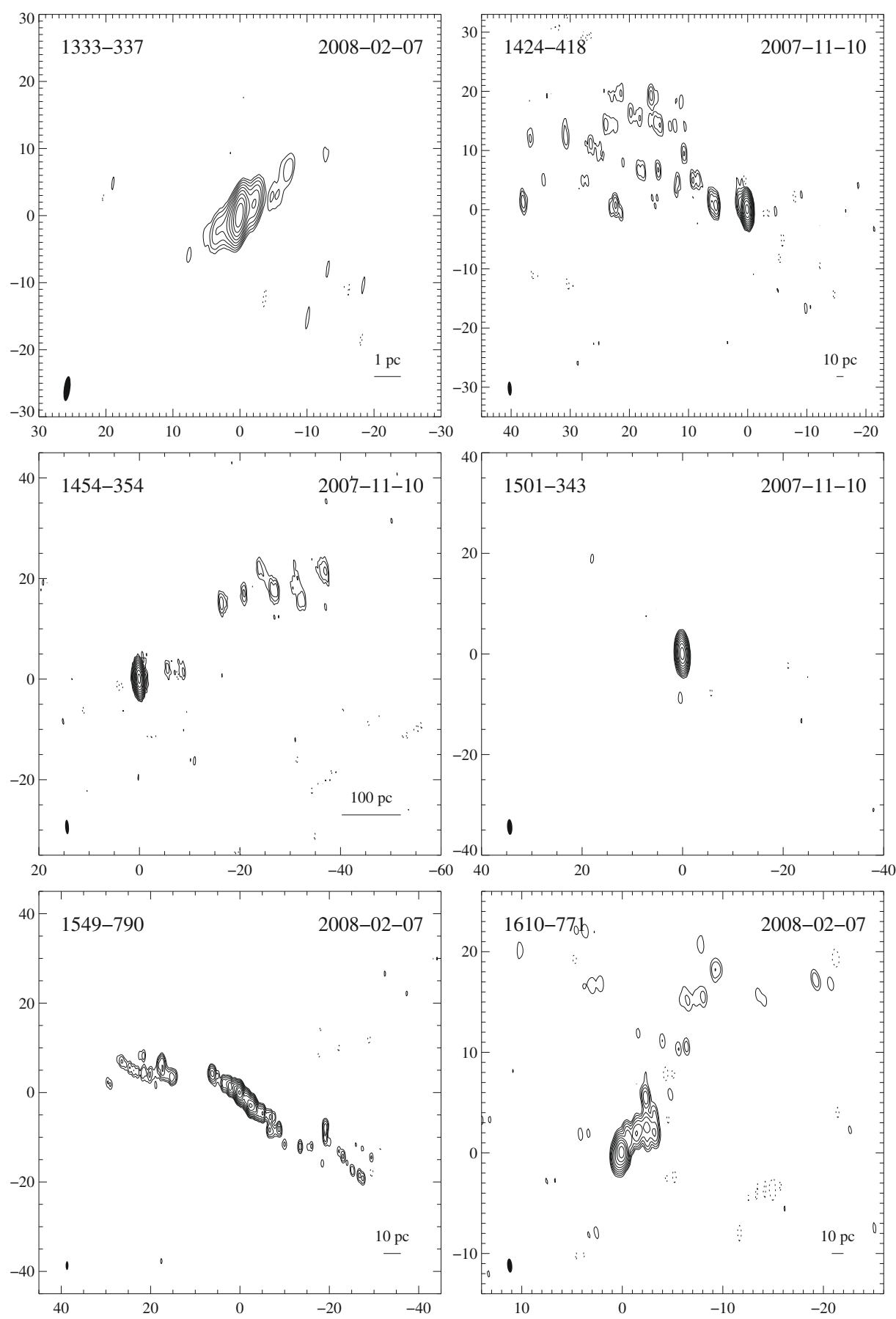

Fig. 4. Same as Fig. 1.

to the southeast of the core and reveals an additional component in between, separated from the core by about 7 mas. Tingay et al. (2002) present a component which is located about 1 mas to the eastsoutheast of the core. Our image has less resolution because only Australian antennas participated in this observation, but it does pick up more extended emission than previous images, revealing an extremely large continuous jet out to more than 50 mas to the south-east.

0454-463 This flat spectrum radio source (Kuehr et al. 1981) is a highly polarized quasar. Impey \& Tapia (1990) measured a polarization of $7.1 \%$ and Wills et al. (1992) even $27.1 \%$. The source was detected with EGRET (Thompson et al. 1993a) but somewhat surprisingly (given that it is a very strong radio source with flux over $3.6 \mathrm{Jy}$ at $8.4 \mathrm{GHz}$ ) it has not been detected by the LAT as a bright $\gamma$-ray source in the 3 -month LAT data (Abdo et al. 2009b). The VLBI image of 0454-463 by Ojha et al. (2004a) shows a compact core without additional components. Our image also shows a bright compact core with some very faint extended emission out to $\sim 25$ mas to the east. The tapered image (Fig. 9) does show this eastern jet emission more clearly but does not reveal any additional emission on larger scales.

0506-612 This source has been classified as a low-confidence potential identification of the EGRET source 3EG J0512-6150 by Hartman et al. (1999) and as a plausible identification by 

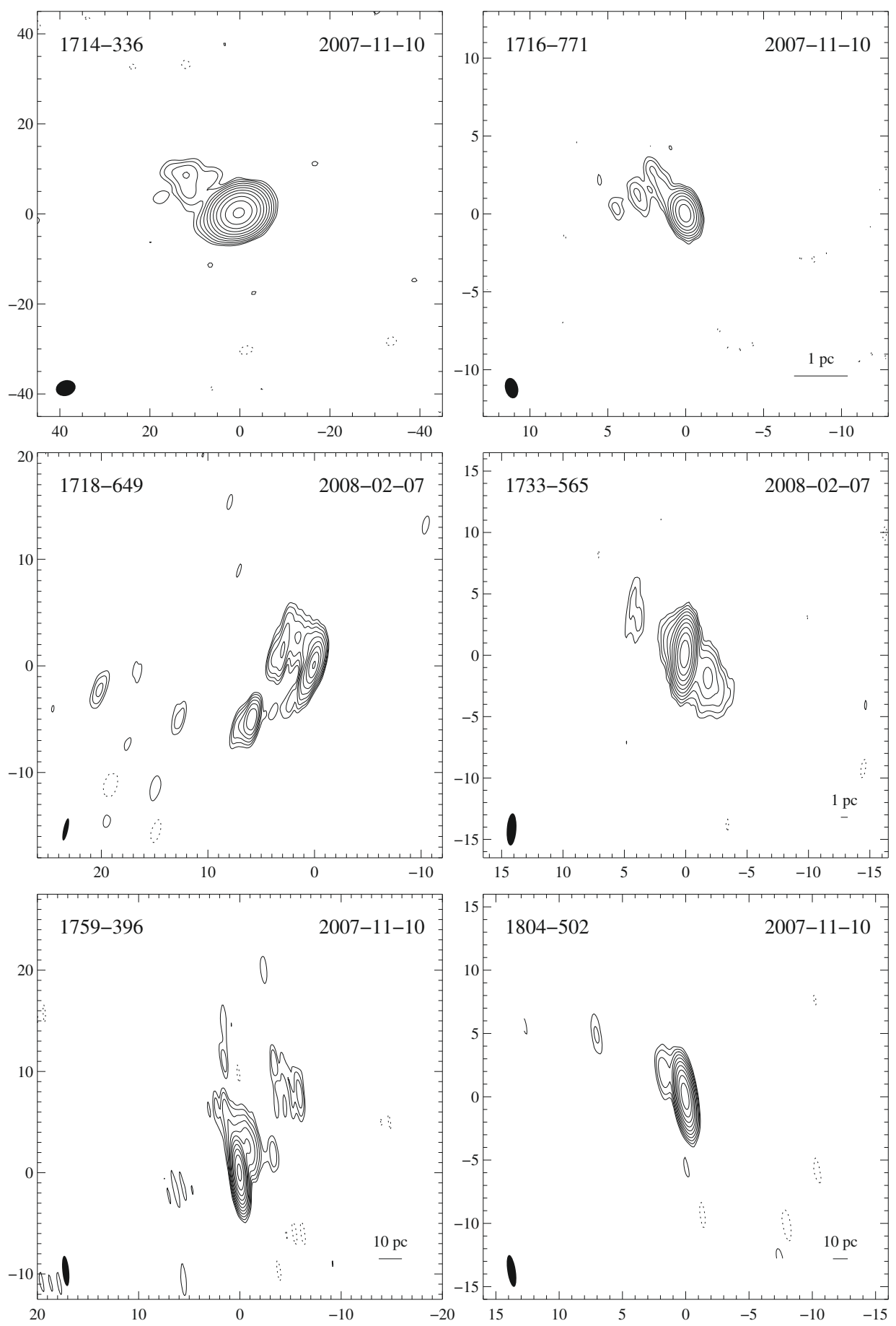

Fig. 5. Same as Fig. 1.

Mattox et al. (2001). Ojha et al. (2004a) find an unresolved source. Our image (Fig. 2) reveals a compact jet component at $\sim 2$ mas north-west of the core and a fainter jet towards the west-north-west. Our tapered image in Fig. 9 reveals more extended jet emission turning to the west-south-west on scales $\sim 30-80$ mas from the core, which was heavily resolved in the naturally-weighted image.

0518-458 Pictor A is one of the closest, powerful FR II type radio galaxies (Fanaroff \& Riley 1974). Its strong doublelobed radio structure is oriented along the east-west direction (Christiansen et al. 1977). X-ray emission from the nucleus, the jet to the west of the nucleus, the western radio hot spot, and the eastern radio lobe was detected with Chandra (Wilson et al. 2001). Tingay et al. (2000) found subluminal motions in the western jet. No parsec-scale counterjet has been detected so far. Our image shows emission from the western jet out to about 35 mas from the core, with at least three compact jet components in the inner 15 mas, which may be associated with the components C 2, C 3, and C 4 seen by Tingay et al. (2000), in which case we would derive speeds of $\sim 0.15 \mathrm{mas} \mathrm{yr}^{-1}$ for all three components. No counterjet emission is seen in the image shown in Fig. 2, but we note that limitations in the $(u, v)$-coverage do not allow us to put a strong upper limit on the brightness of a potential counterjet. 
A\&A 519, A45 (2010)
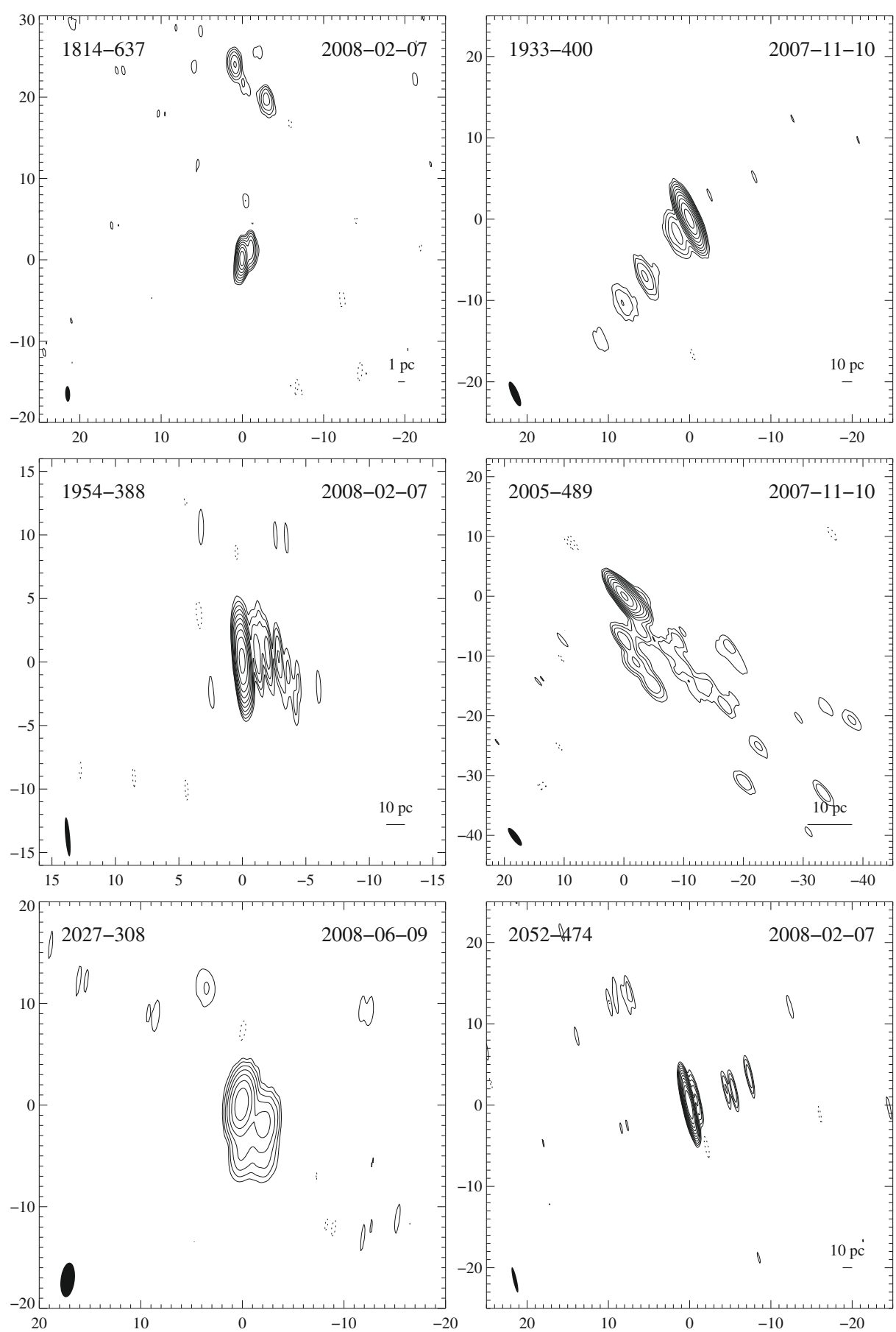

Fig. 6. Same as Fig. 1.

0521-365 This source shows one of the best examples of an optical synchrotron jet (Danziger et al. 1979; Boisson et al. 1989; Scarpa et al. 1999). It possesses strong extended radio and X-ray emission in addition to a bright compact radio source and there are broad and variable nuclear optical emission lines (Ulrich 1981; Scarpa et al. 1995). The VLBI map of this source at $5 \mathrm{GHz}$ by Shen et al. (1998b) shows a core jet northwestward consisting of a core and two additional components separated from the core by $\sim 3.4$ mas and $\sim 8.3$ mas. High-resolution VLBI images of this source have also been presented by Tingay et al. (1996a) and Tingay \& Edwards (2002) but the TANAMI image shown in Fig. 2 shows the parsec-scale jet of this nearby active galaxy with unprecedented resolution and image fidelity.
0537-441 This source has a GPS spectrum peaking at $5 \mathrm{GHz}$ (Tornikoski et al. 2001) and has been a strong variable EGRET gamma-ray source (Thompson et al. 1993b; Hartman et al. 1999). It is known to be a strong source at all wavelengths (Pian et al. 2002). It has been detected by the LAT in a flaring state weeks after the beginning of Fermi science operations (Tosti 2008), and it is one of the two brightest blazars in the southern $\gamma$-ray sky so far (Abdo et al. 2009b). A VLA image by Cassaro et al. (1999) shows a curved jet-like structure leading to the west. VLBI images at $2.3 \mathrm{GHz}$ and $8.4 \mathrm{GHz}$ for several epochs are available in the United States Naval Observatory's Radio Reference Frame Image Database at (RRFID; http://www. usno.navy.mil/RRFID/). At both frequencies the RRFID 

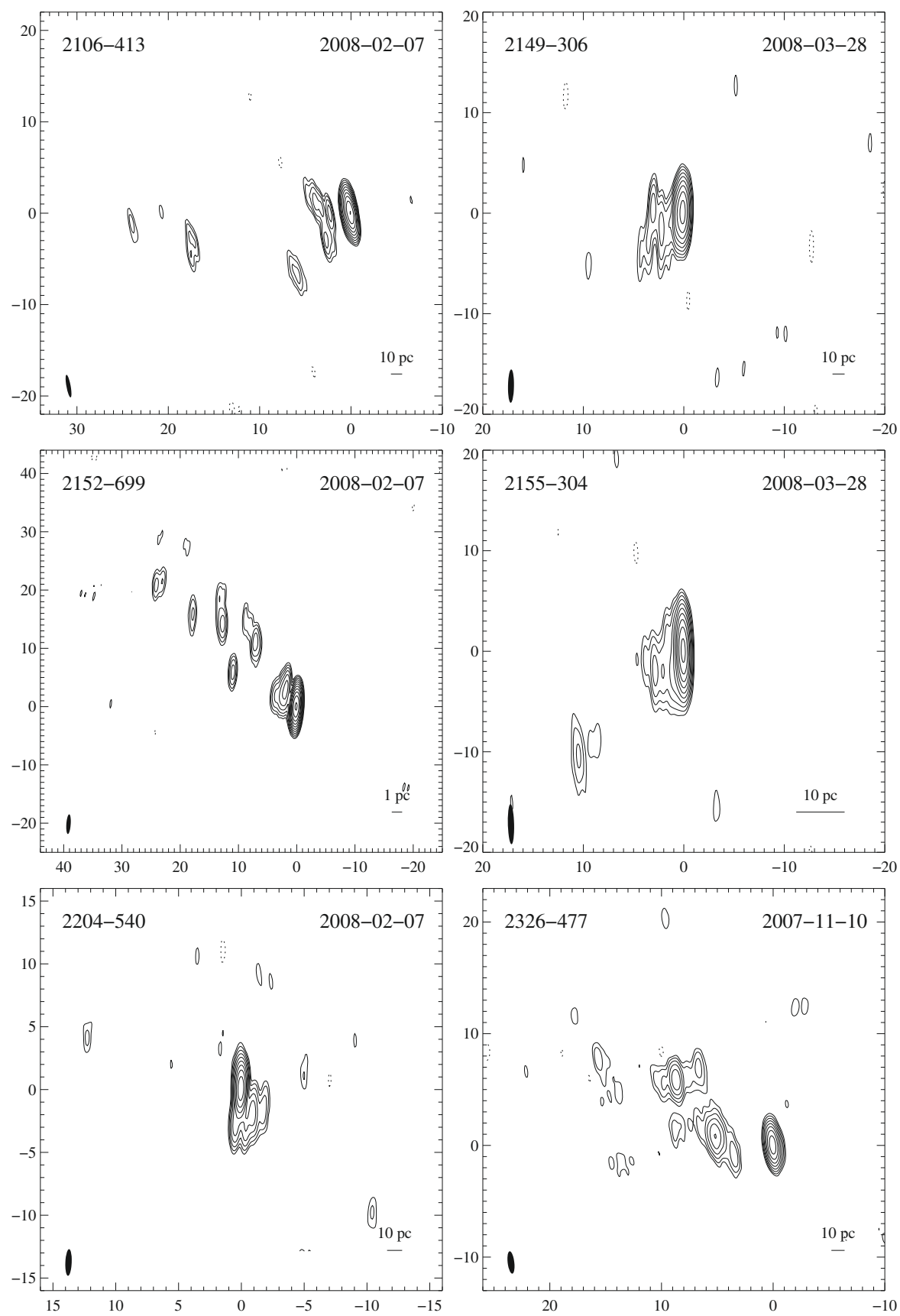

Fig. 7. Same as Fig. 1.

images show a strong core with significant emission a few milliarcseconds to the northeast. This is the same morphology as seen in our TANAMI image. We find an unusually high brightness temperature exceeding $10^{14} \mathrm{~K}$ of the core which is both very compact (although slightly resolved) and very bright.

0625-354 This source exhibits a FR I radio-galaxy morphology but its optical spectrum is more similar to a BL Lac object (Wills et al. 2004). The optical counterpart is a giant elliptical in the center of the cluster Abell 3392 and exhibits a strong point source nucleus (Govoni et al. 2000). The VLBA map of this source by Fomalont et al. (2000) shows a faint component to the southeast of the core, which is consistent with the direction of the larger-scale jet. A $2.3 \mathrm{GHz}$ image obtained by Venturi et al. (2000) shows a diffuse outer jet component at $\sim 200$ mas south-east of the core. Our tapered image (Fig. 9) shows jet emission on intermediate scales out to $\sim 95$ mas from the core.

0637-752 Yaqoob et al. (1998) find a peculiar emission line in the X-ray spectrum of this radio-loud quasar with an energy of $1.60 \pm 0.07 \mathrm{keV}$ and equivalent width $59_{-34}^{+38} \mathrm{eV}$ in the quasar frame. This source was the first Chandra target, a $100 \mathrm{kpc}$ X-ray jet to the west coinciding with the optical and radio contours was discovered and at least 4 knots in the jet were resolved, which were separated from the core by a few arcseconds (Schwartz et al. 2000; Chartas et al. 2000). The VLBI image by Tingay et al. (2002) shows a component about 1.5 mas to the west of the core. Ojha et al. (2004a) find a component located 5 mas from the core. Edwards et al. (2006) observed superluminal motion of this jet in the parsec-scale. The TANAMI image confirms the 


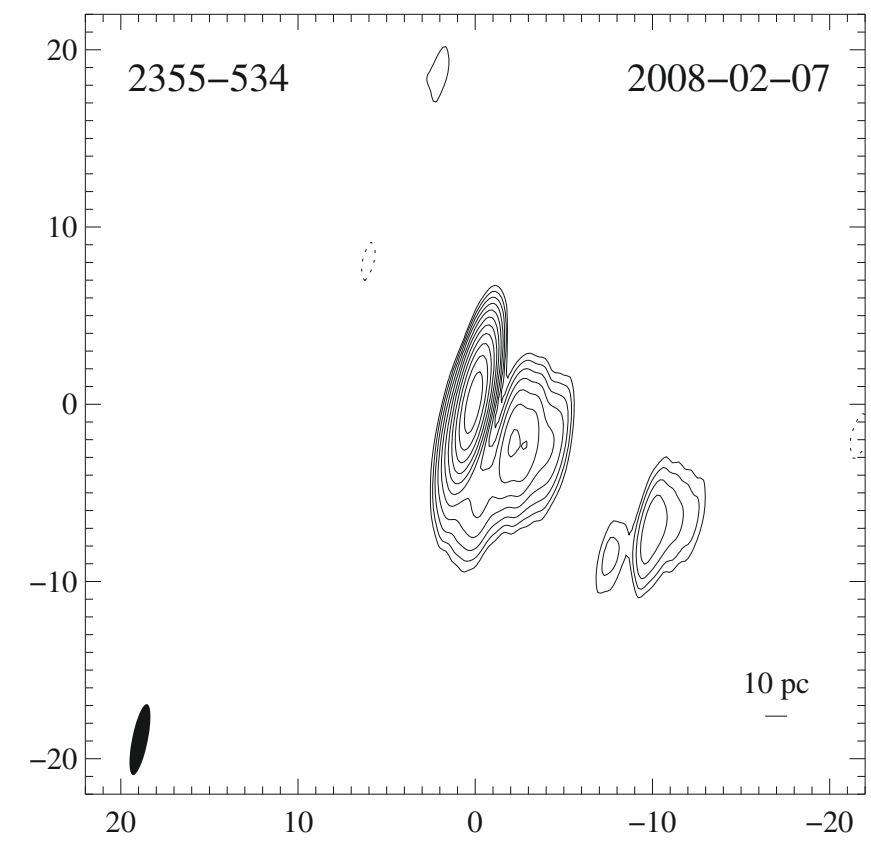

Fig. 8. Same as Fig. 1.

structure seen in these earlier images, revealing a jet out to about 10 mas in the same direction and significant components beyond that. The tapered image (Fig. 9) shows that the jet emission extends beyond 50 mas from the core. Despite being a strong superluminal blazar this source was not detected by EGRET, and has not yet been detected by the LAT (Abdo et al. 2009b).

1104-445 The first southern VLBI Experiment observations (Preston et al. 1989) show a component located about 17 mas to the eastnortheast (position angle $75^{\circ}$ ) of the compact core of this source. Shen et al. (1997) find a jet to the north-east with curvature from the northeast to north at $\sim 1.8$ mas. Tingay et al. (2002) present a highly resolved map, revealing structure in the northeast and southwest. In the TANAMI image (Fig. 3), there is one jet component $\sim 3$ mas north-east of the core, which is spread over $>8$ mas in the north-south direction. This structure may represent a wide opening angle of the jet rather than curvature as suggested by Shen et al. (1997).

1144-379 This source has been classified as a BL Lac object, due to optical, infrared and rapid radio variability, and its featureless powerlaw spectrum (Nicolson et al. 1979) but Veron-Cetty \& Veron (2006) list it as a quasar. The VLBI map by Shen et al. (1997) showed an unresolved core. Several RRFID epochs also show an unresolved core at $8.4 \mathrm{GHz}$ but a few show an extension to the southwest. The TANAMI image shows a clear jet in the same direction to about 16 mas along with significant emission at about 30 mas from the core better revealed in the tapered image (Fig. 10). This bright, rapidly variable source was not detected by EGRET but has already been detected by the LAT (Abdo et al. 2009b).

1257-326 This source is a flat-spectrum, radio-loud quasar, which shows extreme intra-day variability due to interstellar scintillation (Bignall et al. 2003, 2006). No VLBI image of this source has been published so far. The TANAMI image shows a well-collimated but possibly transversally resolved jet out to about 30 mas north-west of the core. Because of its relatively low signal-to-noise ratio, the core appears unresolved but the limit on its brightness temperature is relatively low, only $10^{11} \mathrm{~K}$.

1313-333 The spectrum of this quasar is extremely flat and the source, which is very variable at high radio frequencies, has been associated with the EGRET source 3EG J1313-431 (Nolan et al. 1996; Tornikoski et al. 2002). VLBA images at 2.32 and $8.55 \mathrm{GHz}$ by Fey et al. (1996) show jet components to the west, separated from the core by 4.7 mas at $2.32 \mathrm{GHz}$, and 0.9 and 4.5 mas at $8.55 \mathrm{GHz}$, respectively. Our image shows emission in the same direction and on the same scales but at higher resolution than published before.

1322-428 Centaurus A is the nearest AGN and is one of only two radio galaxies detected by Fermi in its first three months of observation (Abdo et al. 2009b). This very well studied source was also detected in $\gamma$-rays by EGRET. The TANAMI image shows a long collimated jet extending to the northwest as well as a weak counterjet. The counterjet of Cen A was also seen in past VLBI images (e.g., Horiuchi et al. 2006).

1323-526 This is a bright but relatively poorly studied optically unclassified object. It shows intra-day variability (McCulloch et al. 2005). Bignall et al. (2008) suggest a tentative association of this flat-spectrum IDV radio source with the unidentified EGRET source 3EGJ1316-5244. The TANAMI image shows a continuous jet extending about 8 mas to the south of the core.

1333-337 (IC 4296) A symmetric jet and counterjet system is visible in the kpc regime, which is orientated in the northwestsoutheast direction. The outer lobes of the jets are separated by about 30 arcmin (Goss et al. 1977; Killeen et al. 1986). The central nuclear luminosity is relatively weak compared to "normal" radio-loud AGN (Pellegrini et al. 2003). This galaxy was not detected by EGRET, nor has it been detected by the LAT in its first three months. The TANAMI image shows its parsec-scale structure to have a jet and a counterjet aligned northwest-southeast i.e. the same orientation as its kiloparsec structure.

1424-418 Previous VLBI images of this highly optically polarized quasar (Impey \& Tapia 1988) showed jet components in different directions. Preston et al. (1989) found a component to the northeast separated by 23 mas from the core, while the image by Shen et al. (1998b) showed a component about 3 mas to the northwest of the core. The VSOP image by Tingay et al. (2002) shows a northeastward extension of the core and the one by Ojha et al. (2004a) reveals weak structure in the same direction. Our TANAMI image is in agreement with the results of Preston et al. (1989); Tingay et al. (2002); Ojha et al. (2004a) but reveals substantially more detail than these previous images. The jet extends out to about 40 mas east from the core and is very diffuse and resolved, best revealed in the tapered image in Fig. 10.

1454-354 This flat-spectrum radio quasar is not very well studied. This source was included in our initial TANAMI sample as a possible counterpart for the EGRET source 3EG J1500-3509 (with 1501-343 being an alternative association; Mattox et al. 2001; Sowards-Emmerd et al. 2004). This 
R. Ojha et al.: TANAMI first epoch $8.4 \mathrm{GHz}$ images
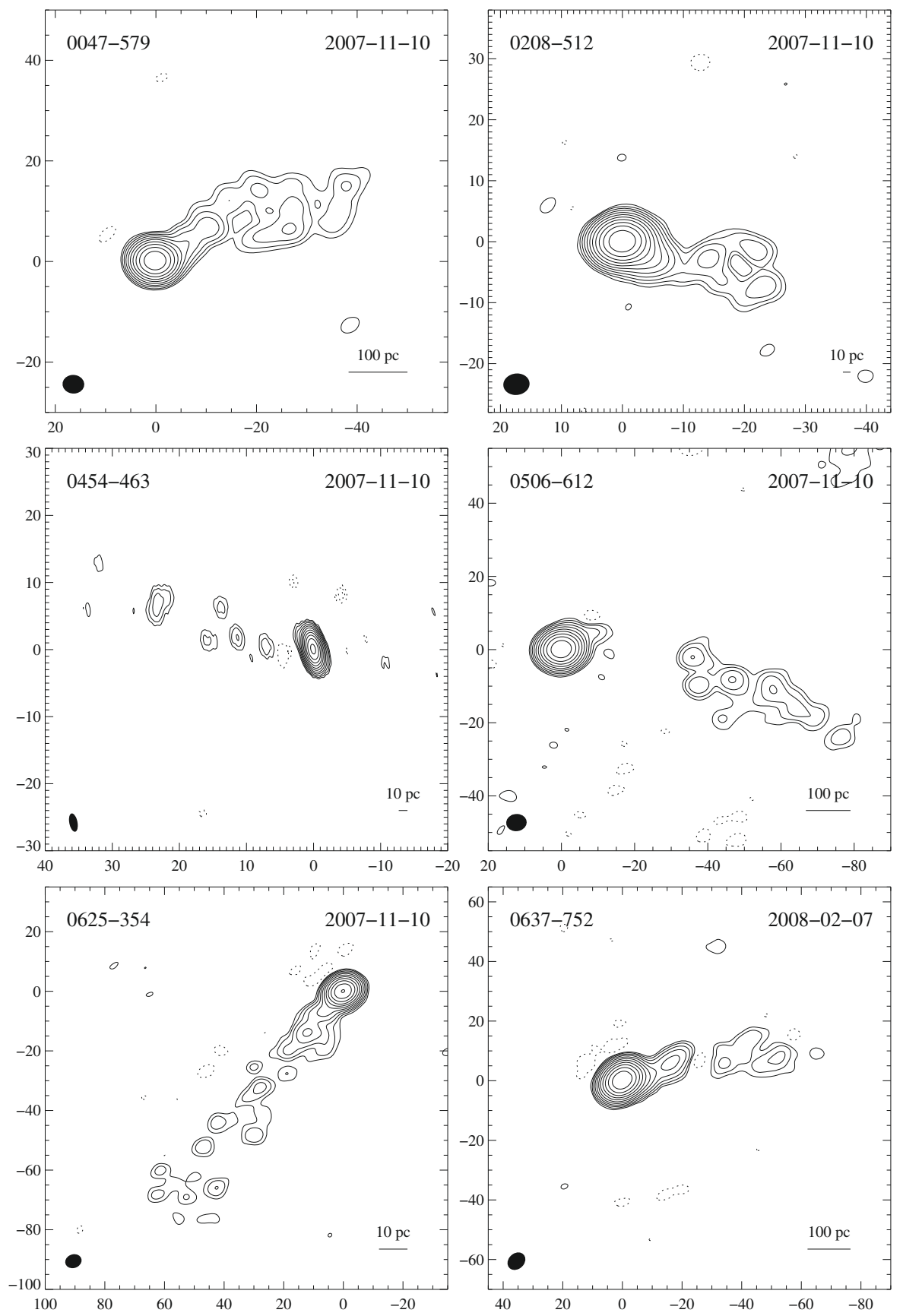

Fig. 9. Tapered contour maps of 13 TANAMI sources at $8.4 \mathrm{GHz}$ revealing more extended emission than visible in the naturally-weighted images. The scale of each image is in milliarcseconds. The FWHM Gaussian restoring beam applied to the images is shown as a hatched ellipse in the lower left of each panel. Each panel also shows a bar representing a linear scale of $10 \mathrm{pc}$ or $100 \mathrm{pc}$ except for the sources without a measured redshift.

was the first $\gamma$-ray blazar to be detected in outburst by Fermi (Marelli 2008). An analysis of the Fermi data from this outburst are presented in Abdo et al. (2009c), to which we contributed the first deep high-resolution VLBI image of this source (naturally weighted and tapered images reproduced in Figs. 4 and 10). These images show a bright compact core with some diffuse emission extending northwest to about 40 mas.

1501-343 Along with 1454-354, this source has been suggested by Sowards-Emmerd et al. (2004) as a possible blazar counterpart for 3EGJ1500-3509. The early detection of 1454-354 by the LAT makes 1501-343 the more unlikely association of the 3EG source. Petrov et al. (2007) did not detect this source at $22 \mathrm{GHZ}$ in six observations with a minimum flux density limit of $170 \mathrm{mJy}$. TANAMI presents the first VLBI image of this source, revealing a very compact structure with only a very weak possible extension to the south. Despite the relatively low core flux density, the brightness temperature exceeds $5 \times 10^{12} \mathrm{~K}$.

1549-790 This luminous narrow-line radio galaxy has been suggested to be an object at an early stage of its evolution (Tadhunter et al. 2001) and seems to be accreting at close to the Eddington rate (Holt et al. 2006) possibly related to a recent 

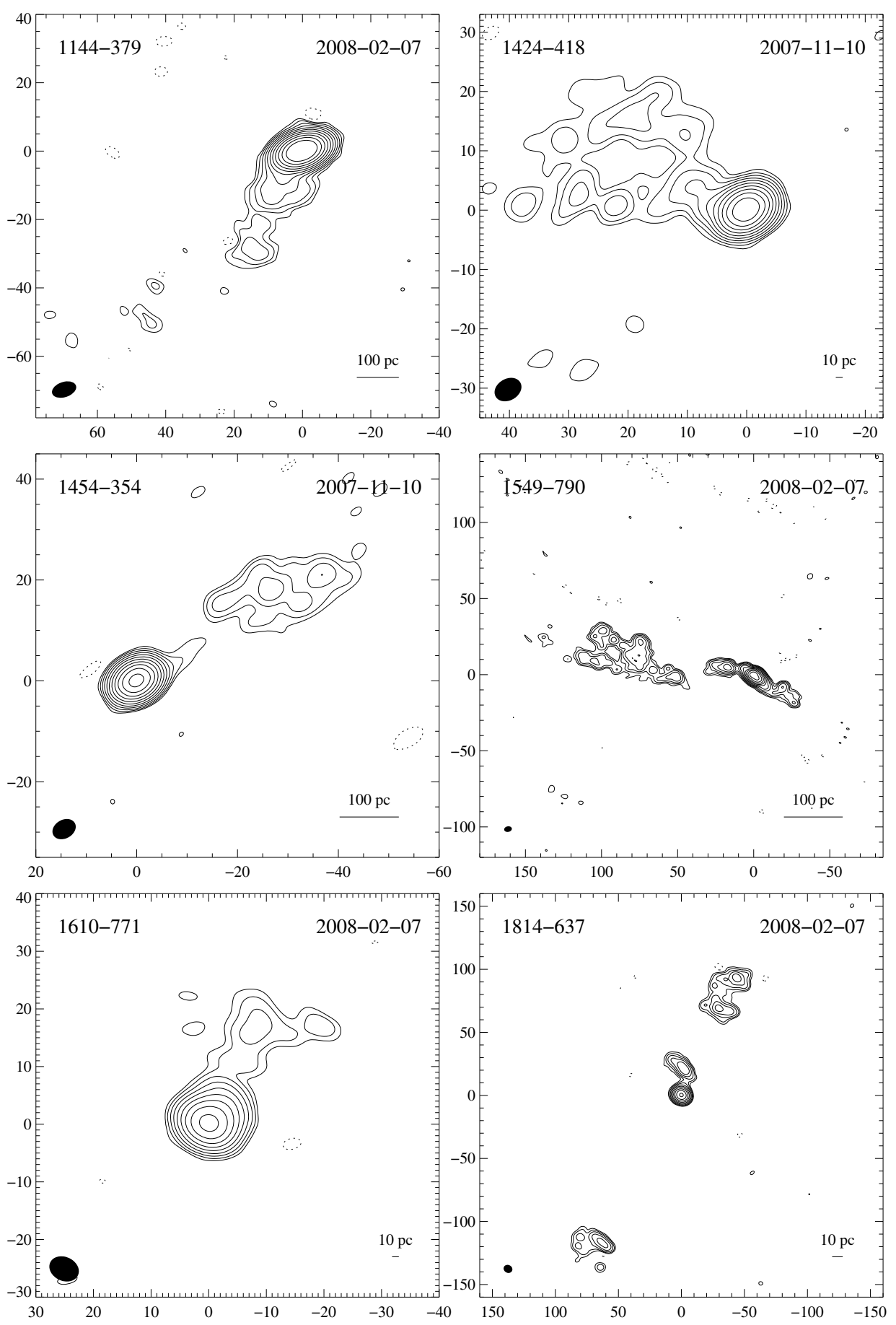

Fig. 10. Same as Fig. 9.

merger. Previously, a one-sided core-jet type structure has been reported for this source (Holt et al. 2006), which led to difficulties in understanding the absence of broad emission lines and a strong non-stellar optical continuum, as well as the presence of $\mathrm{HI}$ absorption (Morganti et al. 2001). The tapered TANAMI image shown in Fig. 10 shows a symmetric inner system with a jet and a pronounced counterjet extending out to about 30 mas east and west of the brightest feature, which we tentatively identify as the core. At larger distances towards the west, there is a very large and diffuse emission region ranging from $\sim 50$ mas to $\sim 120-150$ mas from the core. There is an emission gap of about 10 mas between the inner and outer eastern structure and an offset in declination may indicate jet curvature. Compared with the images presented by Holt et al. (2006), this image is much deeper. The brightness distribution of the eastern outer structure is similar but not the same as the one in Holt et al. (2006). The difference could be due to the better surface-brightness sensitivity in our image or due to image-deconvolution uncertainties. The inner structure is shown in full resolution (natural weighting) in Fig. 4. Immediately to the west of the brightest central component, which we identify as the core of the jet, there is a region of relatively low flux density. The first bright feature in the counterjet seems to be located $\sim 4$ mas to the west and may be interpreted as the base of the counterjet. The central region between the two cores may then be attenuated in brightness because of free-free absorption in a central obscuring torus 


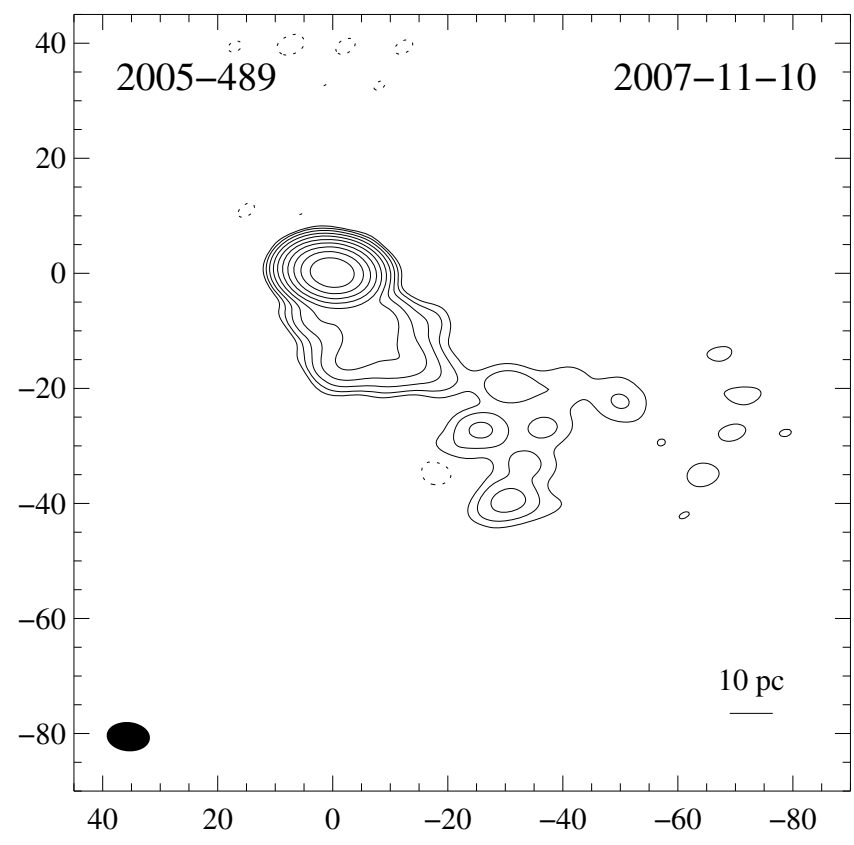

Fig. 11. Same as Fig. 9.

similar to the one in NGC 1052 (e.g., Kadler et al. 2004, and references therein). This will be addressed in a future paper considering spectral information from combined $8.4 \mathrm{GHz}$ and $22 \mathrm{GHz}$ TANAMI data.

1610-771 Using data with 22 mas resolution Preston et al. (1989) modeled this source as a 3.8 Jy component 10 mas in extent with a $1.4 \mathrm{Jy}$ halo approximately 50 mas in diameter. The VSOP image by Tingay et al. (2002) revealed the small scale structure within $\sim 1$ mas from the core to consist of 3 components at a position angle of -30 degree with no obvious identification of the core. Our image shows a curved jet towards the northeast, extending about 5 mas from the core and diffuse emission on larger scales to the north. The tapered image in Fig. 10 shows this diffuse jet to extend up to about 25 mas north of the core.

1714-336 This is a possible counterpart of 3EG J1718-3313 (Sowards-Emmerd et al. 2004) that has not yet been detected by the LAT (Abdo et al. 2009b). The TANAMI image of this BL Lac appears to be the first at VLBI resolution and shows a bright core with a jet extending over 20 mas to the northeast. Only Australian antennas participated in this observation so that the image resolution is worse than for most other TANAMI images presented in this paper. The core brightness temperature is unusually low.

1716-771 Tornikoski et al. (2002) suggested this unclassified source of undetermined redshift as a possible counterpart for 3EG J1720-7820 but within the first three months of Fermi allsky observations, it did not show up as a bright $\gamma$-ray source (Abdo et al. 2009b). This is one of the faintest sources in the TANAMI sample with no previous VLBI image. Figure 5 shows an unresolved compact core of relatively high brightness temperature $T_{\mathrm{B}}>7 \times 10^{10}$. There is a faint jet extending about 4 mas to the northeast.
1718-649 With a distance of $56 \mathrm{Mpc}$ this is one of the closest and best studied GPS sources (e.g. Tingay et al. 2003a). Its structure strengthens the assumption that GPS sources arise as a consequence of galaxy merger activity (Tingay et al. 1997). Tingay \& de Kool (2003) suggest synchrotron self-absorption or free-free absorption are the only possible processes responsible for the gigahertz-peaked spectrum. The maps by Tingay et al. (2002) and Ojha et al. (2004a) show two components separated by about 7 mas. It was not possible to identify which one corresponds to the core. The map by Preston et al. (1989) also showed a double component aligned in the same direction (southeast to northwest). Our TANAMI image shows two bright components with similar alignment and separation but the northwestern component is clearly more "core-like" in appearance. There is also a bright extension to the northeast of the "core". We classify this source as morphologically irregular.

1733-565 This FR II radio galaxy has two extended lobes to the southwest and northeast of the core, which are separated by 4.57 arcmin (Hunstead et al. 1982), in between there is bridge and core emission. Bryant \& Hunstead (2002) found rotating emission-line gas extended perpendicular to the radio axis. The VLBI map by Ojha et al. (2004a) shows a compact core, without additional components. The TANAMI image shows a jet as well as a counter jet aligned northeast and southwest i.e. in the same direction as the large scale structure.

1759-396 This source is a possible counterpart of 3EG J1800-3955 (Sowards-Emmerd et al. 2004) and is a low confidence detection with the LAT (Abdo et al. 2009b). The TANAMI image shows a fairly typical core jet source with the jet extending northwest to about 10 mas.

1804-502 This source is a candidate counterpart for 3EG J1806-5005 (Tornikoski et al. 2002) but it has not been a bright $\gamma$-ray source during the first three months of Fermi observations (Abdo et al. 2009b). There is no high-resolution VLBI image of this source in the literature. The TANAMI image shows an unresolved high brightness-temperature core $\left(T_{\mathrm{B}}>8.9 \times 10^{12} \mathrm{~K}\right.$ and a weak jet to the north-west.

1814-637 This is a compact steep spectrum (CSS) source with three components oriented along a south-southeast to northnorthwest axis. Tzioumis et al. (2002) imaged this source at $2.3 \mathrm{GHz}$ and found two strong components separated by more than 200 mas and a weak $(15 \sigma)$ component in between. Ojha et al. (2004a) found an unresolved core and an additional component about 90 mas to the north-northwest at $8.4 \mathrm{GHz}$ but the alignment between these two images and the identification of the core was not obvious. Our widefield tapered image in Fig. 10 solves this problem. In total, we detect three main components, of which the southernmost and the northernmost can be identified by the outer structures seen by Tzioumis et al. (2002). Apparently, Ojha et al. (2004a) did not pick up the emission from the southern component. The central feature in our image represents the core of the source with a parsec-scale jet extending to the north, whose fine scale structure can best be seen in the naturally weighted image in Fig. 6. The comparison with the $2.3 \mathrm{GHz}$ image by Tzioumis et al. (2002) suggests that the core component may have an inverted spectrum and could be affected by free-free absorption, although clearly a simultaneous 
Table 3. Source list.

\begin{tabular}{|c|c|c|c|c|c|c|c|c|c|c|}
\hline Source ${ }^{a}$ & Name $^{b}$ & $\begin{array}{c}\mathrm{RA} \\
(\mathrm{J} 2000.0)^{c}\end{array}$ & $\begin{array}{c}\text { Dec } \\
(\mathrm{J} 2000.0)^{c}\end{array}$ & $\mathrm{ID}^{d}$ & $\begin{array}{c}\text { Magnitude } \\
V^{e}\end{array}$ & $\begin{array}{c}\text { Redshift } \\
z^{f}\end{array}$ & $\begin{array}{c}\text { Radio } \\
\text { Sample } \\
\end{array}$ & $\begin{array}{c}\gamma \\
\text { Sample }\end{array}$ & EGRET $^{g}$ & $\mathrm{LAT}^{\prime}$ \\
\hline $0047-579$ & & $00^{\mathrm{h}} 49^{\mathrm{m}} 59^{\mathrm{s}} .4731$ & $-57^{\circ} 38^{\prime} 27^{\prime \prime} .339$ & $\mathrm{Q}$ & 18.50 & $1.797^{1}$ & $\mathrm{Y}$ & $\mathrm{N}$ & $\mathrm{N}$ & $\mathrm{N}$ \\
\hline $0208-512$ & & $02^{\mathrm{h}} 10^{\mathrm{m}} 46^{\mathrm{s}} .2004$ & $-51^{\circ} 01^{\prime} 02^{\prime \prime} 891$ & B & 16.93 & $0.99^{2}$ & $\mathrm{Y}$ & $\mathrm{Y}$ & $\mathrm{Y}$ & $\mathrm{Y}$ \\
\hline $0332-403$ & & $03^{\mathrm{h}} 34^{\mathrm{m}} 13^{\mathrm{s}} .6544$ & $-40^{\circ} 08^{\prime} 25^{\prime \prime} 396$ & $\mathrm{~B}$ & 18.50 & $1.445^{3}$ & $\mathrm{Y}$ & $\mathrm{N}$ & $\mathrm{N}$ & $\mathrm{Y}$ \\
\hline $0405-385$ & & $04^{\mathrm{h}} 06^{\mathrm{m}} 59^{\mathrm{s}} .0353$ & $-38^{\circ} 26^{\prime} 28^{\prime \prime} 042$ & Q & 18.00 & $1.285^{4}$ & $\mathrm{~N}$ & $\mathrm{~N}$ & $\mathrm{~N}$ & $\mathrm{Y}^{*}$ \\
\hline $0438-436$ & & $04^{\mathrm{h}} 40^{\mathrm{m}} 17^{\mathrm{s}} .1799$ & $-43^{\circ} 33^{\prime} 08^{\prime \prime} 602$ & Q & 18.8 & $2.863^{5}$ & $\mathrm{Y}$ & $\mathrm{N}$ & $\mathrm{N}$ & $\mathrm{N}$ \\
\hline $0454-463$ & & $04^{\mathrm{h}} 55^{\mathrm{m}} 50^{\mathrm{s}} .7724$ & $-46^{\circ} 15^{\prime} 58^{\prime \prime} 681$ & $\mathrm{Q}$ & 17.40 & $0.8528^{6}$ & $\mathrm{Y}$ & $\mathrm{Y}$ & $\mathrm{Y}$ & $\mathrm{N}$ \\
\hline $0506-612$ & & $05^{\mathrm{h}} 06^{\mathrm{m}} 43^{\mathrm{s}} .9887$ & $-61^{\circ} 09^{\prime} 40.993$ & Q & 16.85 & $1.093^{3}$ & $\mathrm{~N}$ & $\mathrm{Y}$ & $\mathrm{C}$ & $\mathrm{N}$ \\
\hline $0518-458$ & Pictor A & $05^{\mathrm{h}} 19^{\mathrm{m}} 49^{\mathrm{s}} .69$ & $-45^{\circ} 46^{\prime} 44^{\prime \prime} 5$ & $\mathrm{G}$ & 16.45 & $0.035058^{7}$ & $\mathrm{~N}$ & $\mathrm{~N}$ & $\mathrm{~N}$ & $\mathrm{~N}$ \\
\hline $0521-365$ & ESO 362-G 021 & $05^{\mathrm{h}} 22^{\mathrm{m}} 57^{\mathrm{s}} .9846$ & $-36^{\circ} 27^{\prime} 30^{\prime} 848$ & $\mathrm{~B}$ & 14.50 & $0.055338^{8}$ & $\mathrm{Y}$ & $\mathrm{Y}$ & $\mathrm{C}$ & $\mathrm{N}$ \\
\hline $0537-441$ & & $05^{\mathrm{h}} 38^{\mathrm{m}} 50^{\mathrm{s}} .3614$ & $-44^{\circ} 05^{\prime} 08^{\prime \prime} 934$ & Q & 15.50 & $0.894^{1}$ & $\mathrm{Y}$ & $\mathrm{Y}$ & $\mathrm{Y}$ & $\mathrm{Y}$ \\
\hline $0625-354$ & $\mathrm{OH}-342$ & $06^{\mathrm{h}} 27^{\mathrm{m}} 06^{\mathrm{s}} .72$ & $-35^{\circ} 29^{\prime} 15^{\prime \prime} .4$ & G & 16.50 & $0.054594^{9}$ & $\mathrm{Y}$ & $\mathrm{N}$ & $\mathrm{N}$ & $\mathrm{N}$ \\
\hline $0637-752$ & & $06^{\mathrm{h}} 35^{\mathrm{m}} 46^{\mathrm{s}} .5079$ & $-75^{\circ} 16^{\prime} 16^{\prime \prime} 814$ & Q & 15.75 & $0.653^{10}$ & $\mathrm{Y}$ & $\mathrm{N}$ & $\mathrm{N}$ & $\mathrm{N}$ \\
\hline $1104-445$ & & $11^{\mathrm{h}} 07^{\mathrm{m}} 08^{\mathrm{s}} \cdot 6929$ & $-44^{\circ} 49^{\prime} 07^{\prime \prime} 567$ & Q & 18.20 & $1.598^{1}$ & $\mathrm{Y}$ & $\mathrm{N}$ & $\mathrm{N}$ & $\mathrm{N}$ \\
\hline $1144-379$ & & $11^{\mathrm{h}} 47^{\mathrm{m}} 01^{\mathrm{s}} .3706$ & $-38^{\circ} 12^{\prime} 11^{\prime \prime} 022$ & Q & 16.20 & $1.048^{11}$ & $\mathrm{~N}$ & $\mathrm{~N}$ & $\mathrm{~N}$ & $\mathrm{Y}$ \\
\hline $1257-326$ & & $13^{\mathrm{h}} 00^{\mathrm{m}} 42^{\mathrm{s}} .4259$ & $-32^{\circ} 53^{\prime} 12^{\prime \prime} 110$ & Q & 18.70 & $1.256^{12}$ & $\mathrm{~N}$ & $\mathrm{~N}$ & $\mathrm{~N}$ & $\mathrm{~N}$ \\
\hline $1313-333$ & & $13^{\mathrm{h}} 16^{\mathrm{m}} 07^{\mathrm{s}} .9859$ & $-33^{\circ} 38^{\prime} 59^{\prime} \cdot 171$ & Q & 20.00 & $1.21^{13}$ & $\mathrm{~N}$ & $\mathrm{Y}$ & $\mathrm{C}$ & $\mathrm{N}$ \\
\hline $1322-428$ & Cen A, NGC 5128 & $13^{\mathrm{h}} 25^{\mathrm{m}} 27^{\mathrm{s}} .6152$ & $-43^{\circ} 01^{\prime} 08^{\prime \prime} 805$ & $\mathrm{G}$ & 7.84 & $0.001825^{14}$ & $\mathrm{Y}$ & $\mathrm{Y}$ & $\mathrm{Y}$ & $\mathrm{Y}$ \\
\hline $1323-526$ & PMN J1326-5256 & $13^{\mathrm{h}} 46^{\mathrm{m}} 48^{\mathrm{s}} .70$ & $-52^{\circ} 56^{\prime} 22^{\prime \prime} 0$ & $\mathrm{U}$ & $\ldots$ & $\ldots$ & $\mathrm{N}$ & $\mathrm{Y}$ & $\mathrm{C}^{30}$ & $\mathrm{~N}$ \\
\hline $1333-337$ & IC 4296 & $13^{\mathrm{h}} 36^{\mathrm{m}} 39^{\mathrm{s}} .05$ & $-33^{\circ} 57^{\prime} 57^{\prime \prime} .2$ & G & 11.61 & $0.012465^{15}$ & $\mathrm{~N}$ & $\mathrm{~N}$ & $\mathrm{~N}$ & $\mathrm{~N}$ \\
\hline $1424-418$ & & $13^{\mathrm{h}} 27^{\mathrm{m}} 56^{\mathrm{s}} .2975$ & $-42^{\circ} 06^{\prime} 19 ! \cdot 437$ & Q & 17.7 & $1.522^{16}$ & $\mathrm{Y}$ & $\mathrm{Y}$ & Y & $\mathrm{N}$ \\
\hline $1454-354$ & & $14^{\mathrm{h}} 57^{\mathrm{m}} 26^{\mathrm{s}} .7117$ & $-35^{\circ} 39^{\prime} 09^{\prime} 971$ & $\mathrm{Q}$ & 19.50 & $1.424^{17}$ & $\mathrm{Y}$ & $\mathrm{Y}$ & $\mathrm{Y}^{31}$ & $\mathrm{Y}$ \\
\hline $1501-343$ & PMN J1505-3432 & $15^{\mathrm{h}} 05^{\mathrm{m}} 02^{\mathrm{s}} .4$ & $-34^{\circ} 32^{\prime} 57^{\prime \prime} 0$ & $\mathrm{U}$ & $\ldots$ & $\ldots$ & $\mathrm{N}$ & $\mathrm{Y}$ & $\mathrm{Y}^{31}$ & $\mathrm{~N}$ \\
\hline $1549-790$ & & $15^{\mathrm{h}} 56^{\mathrm{m}} 58^{\mathrm{s}} .8697$ & $-79^{\circ} 14^{\prime} 04^{\prime \prime} .281$ & G & 18.50 & $0.1501^{18}$ & $\mathrm{Y}$ & $\mathrm{N}$ & $\mathrm{N}$ & $\mathrm{N}$ \\
\hline $1610-771$ & & $16^{\mathrm{h}} 17^{\mathrm{m}} 49^{\mathrm{s}} .2726$ & $-77^{\circ} 17^{\prime} 18^{\prime \prime} .467$ & Q & 19.00 & $1.71^{19}$ & $\mathrm{Y}$ & $\mathrm{N}$ & $\mathrm{N}$ & $\mathrm{N}$ \\
\hline $1714-336$ & & $17^{\mathrm{h}} 17^{\mathrm{m}} 36^{\mathrm{s}} .0293$ & $-33^{\circ} 42^{\prime} 08^{\prime \prime} .829$ & $\mathrm{~B}$ & & $\ldots$ & $\mathrm{N}$ & $\mathrm{Y}$ & $\mathrm{Y}^{31}$ & $\mathrm{~N}$ \\
\hline $1716-771$ & & $17^{\mathrm{h}} 23^{\mathrm{m}} 50^{\mathrm{s}} .51$ & $-77^{\circ} 13^{\prime} 50^{\prime} 1$ & $\mathrm{U}$ & & & $\mathrm{N}$ & $\mathrm{Y}$ & $\mathrm{C}^{32}$ & $\mathrm{~N}$ \\
\hline $1718-649$ & NGC 6328 & $17^{\mathrm{h}} 23^{\mathrm{m}} 41^{\mathrm{s}} .0296$ & $-65^{\circ} 00^{\prime} 36^{\prime \prime} 615$ & G & 13.16 & $0.014428^{20}$ & $\mathrm{Y}$ & $\mathrm{N}$ & $\mathrm{N}$ & $\mathrm{N}$ \\
\hline $1733-565$ & & $17^{\mathrm{h}} 37^{\mathrm{m}} 35^{\mathrm{s}} .7706$ & $-56^{\circ} 34^{\prime} 03^{\prime \prime} .155$ & $\mathrm{G}$ & 18.00 & $0.098^{21}$ & $\mathrm{Y}$ & $\mathrm{N}$ & $\mathrm{N}$ & $\mathrm{N}$ \\
\hline $1759-396$ & & $18^{\mathrm{h}} 02^{\mathrm{m}} 42^{\mathrm{s}} .680$ & $-39^{\circ} 40^{\prime} 07^{\prime \prime} 905$ & Q & $?$ & $0.296^{22}$ & $\mathrm{~N}$ & $\mathrm{Y}$ & $\mathrm{Y}$ & $\mathrm{Y}^{*}$ \\
\hline $1804-502$ & PMN J1808-5011 & $18^{\mathrm{h}} 08^{\mathrm{m}} 13^{\mathrm{s}} .90$ & $-50^{\circ} 11^{\prime} 54^{\prime \prime} 0$ & $\mathrm{Q}$ & $\ldots$ & $\ldots$ & $\mathrm{N}$ & $\mathrm{Y}$ & $C^{32}$ & $\mathrm{~N}$ \\
\hline $1814-637$ & & $18^{\mathrm{h}} 19^{\mathrm{m}} 35^{\mathrm{s}} .0023$ & $-63^{\circ} 45^{\prime} 48^{\prime \prime} 189$ & G & 18.00 & $0.06270^{23}$ & $\mathrm{~N}$ & $\mathrm{~N}$ & $\mathrm{~N}$ & $\mathrm{~N}$ \\
\hline $1933-400$ & & $19^{\mathrm{h}} 37^{\mathrm{m}} 16^{\mathrm{s}} .2173$ & $-39^{\circ} 58^{\prime} 01^{\prime \prime} 552$ & Q & 18.00 & $0.965^{24}$ & $\mathrm{~N}$ & $\mathrm{Y}$ & $\mathrm{Y}$ & $\mathrm{N}$ \\
\hline $1954-388$ & & $19^{\mathrm{h}} 57^{\mathrm{m}} 59^{\mathrm{s}} .8192$ & $-38^{\circ} 45^{\prime} 06^{\prime \prime} 356$ & Q & 17.70 & $0.63^{25}$ & $\mathrm{Y}$ & $\mathrm{N}$ & $\mathrm{N}$ & $\mathrm{N}$ \\
\hline $2005-489$ & & $20^{\mathrm{h}} 09^{\mathrm{m}} 25^{\mathrm{s}} .3906$ & $-48^{\circ} 49^{\prime} 53^{\prime \prime} 720$ & B & 15.30 & $0.0710^{26}$ & $\mathrm{~N}$ & $\mathrm{Y}$ & $\mathrm{Y}^{33}$ & $\mathrm{Y}$ \\
\hline $2027-308$ & ESO 462-G 027 & $20^{\mathrm{h}} 30^{\mathrm{m}} 57^{\mathrm{s}} .934$ & $-30^{\circ} 39^{\prime} 24^{\prime \prime} 35$ & G & $?$ & - & $\mathrm{N}$ & $\mathrm{Y}$ & $\mathrm{Y}^{31}$ & $\mathrm{~N}$ \\
\hline $2052-474$ & & $20^{\mathrm{h}} 56^{\mathrm{m}} 16^{\mathrm{s}} .3598$ & $-47^{\circ} 14^{\prime} 47^{\prime \prime} 627$ & Q & 19.10 & $1.489^{27}$ & $\mathrm{Y}$ & $\mathrm{Y}$ & $\mathrm{Y}$ & $\mathrm{Y}$ \\
\hline $2106-413$ & & $21^{\mathrm{h}} 09^{\mathrm{m}} 33^{\mathrm{s}} .1885$ & $-41^{\circ} 10^{\prime} 20^{\prime} 605$ & $\mathrm{Q}$ & 21.00 & $1.058^{16}$ & $\mathrm{Y}$ & $\mathrm{N}$ & $\mathrm{N}$ & $\mathrm{N}$ \\
\hline 2149-306 & & $21^{\mathrm{h}} 51^{\mathrm{m}} 55^{\mathrm{s}} .5239$ & $-30^{\circ} 27^{\prime} 53^{\prime \prime} 697$ & Q & 18.40 & $2.345^{3}$ & $\mathrm{~N}$ & $\mathrm{~N}$ & $\mathrm{~N}$ & $\mathrm{~N}$ \\
\hline $2152-699$ & ESO 075-G 041 & $21^{\mathrm{h}} 57^{\mathrm{m}} 05^{\mathrm{s}} .9805$ & $-69^{\circ} 41^{\prime} 23^{\prime \prime} 685$ & $\mathrm{G}$ & 1430 & $0.028273^{28}$ & $\mathrm{~N}$ & $\mathrm{~N}$ & $\mathrm{~N}$ & $\mathrm{~N}$ \\
\hline $2155-304$ & & $21^{\mathrm{h}} 58^{\mathrm{m}} 52^{\mathrm{s}} .0651$ & $-30^{\circ} 13^{\prime} 32^{\prime \prime} 118$ & B & 14.00 & $0.116^{29}$ & $\mathrm{~N}$ & $\mathrm{Y}$ & $\mathrm{Y}$ & $\mathrm{Y}$ \\
\hline $2204-540$ & & $22^{\mathrm{h}} 07^{\mathrm{m}} 43^{\mathrm{s}} .7332$ & $-53^{\circ} 46^{\prime} 33^{\prime \prime} 820$ & Q & 18.00 & $1.206^{3}$ & $\mathrm{Y}$ & $\mathrm{N}$ & $\mathrm{N}$ & $\mathrm{Y}$ \\
\hline $2326-477$ & & $23^{\mathrm{h}} 29^{\mathrm{m}} 17^{\mathrm{s}} .7043$ & $-47^{\circ} 30^{\prime} 19^{\prime} 115$ & Q & 16.79 & $1.2990^{1}$ & $\mathrm{Y}$ & $\mathrm{N}$ & $\mathrm{N}$ & $\mathrm{N}$ \\
\hline $2355-534$ & & $23^{\mathrm{h}} 57^{\mathrm{m}} 53^{\mathrm{s}} .2661$ & $-53^{\circ} 11^{\prime} 13^{\prime \prime} 689$ & Q & 17.80 & $1.0060^{27}$ & $\mathrm{~N}$ & $\mathrm{~N}$ & $\mathrm{~N}$ & $\mathrm{~N}$ \\
\hline
\end{tabular}

Notes. ${ }^{(a)}$ IAU source designation. ${ }^{(b)}$ Alternative source name where appropriate. ${ }^{(c)}$ Right ascension and declination (J2000.0). ${ }^{(d)}$ The optical counterpart, denoted as follows: (G) galaxy, (Q) quasar, (B) BL Lac object, or (U) unclassified. ${ }^{(e)}$ Optical magnitude. ${ }^{(f)}$ Redshift. ${ }^{(g)}$ Y $=$ Detected, $\mathrm{N}=$ Not Detected, $\mathrm{C}=$ Candidate. Those detections and candidates without a reference indicated are from Hartman et al. (1999). (h) Based on the LAT 3-month list. "*” denotes a low confidence detection.

References. (1) Peterson et al. (1976); (2) Wisotzki et al. (2000); (3) Hewitt \& Burbidge (1989); (4) Morton et al. (1978); (5) Stickel et al. (1994); (6) Sulentic et al. (2004); (7) Lauberts \& Valentijn (1989); (8) Keel (1985); (9) Quintana \& Ramirez (1995); (10) Hunstead et al. (1978); (11) Stickel et al. (1989); (12) Perlman et al. (1998); (13) Jauncey et al. (1982); (14) Graham (1978); (15) Smith et al. (2000); (16) White et al. (1988); (17) Jackson et al. (2002); (18) Tadhunter et al. (2001); (19) Hunstead \& Murdoch (1980); (20) HI Parkes All Sky Survey Final Catalog; (21) Tadhunter et al. (1993); (22) Liang \& Liu (2003); (23) Danziger \& Goss (1979); (24) Drinkwater et al. (1997); (25) Browne et al. (1975); (26) Falomo et al. (1987); (27) Jauncey et al. (1984); (28) da Costa et al. (1991); (29) Falomo et al. (1993); (30) Bignall et al. (2008); (31) Sowards-Emmerd et al. (2004); (32) Tornikoski et al. (2002); (33) Lin et al. (1997). 
Table 4. Source structure.

\begin{tabular}{|c|c|c|c|c|c|c|c|c|c|c|c|}
\hline Source & $\begin{array}{c}\text { Epoch } \\
\text { yyyy-mm-dd }\end{array}$ & $\begin{array}{c}\text { Contour }^{a} \\
\left(\mathrm{mJy} \mathrm{beam}^{-1}\right)\end{array}$ & $\begin{array}{c}S_{\text {peak }} \\
\left(\text { Jy beam }^{-1}\right)\end{array}$ & $\begin{array}{r}S_{\text {total }} \\
\text { (Jy) }\end{array}$ & $\begin{array}{c}\theta_{\text {maj }} \\
\text { (mas) }\end{array}$ & $\begin{array}{c}\theta_{\min } \\
(\mathrm{mas})\end{array}$ & $\begin{array}{l}\text { PA } \\
\left({ }^{\circ}\right)\end{array}$ & Structure & $\begin{array}{r}T_{\mathrm{b}}^{b, c} \\
(\mathrm{~K})\end{array}$ & $\begin{array}{l}\text { Core luminosity } \\
\left(\mathrm{W} \mathrm{m}^{-2}\right)\end{array}$ & $\begin{array}{l}\text { Total luminosity } \\
\left(\mathrm{W} \mathrm{m}^{-2}\right)\end{array}$ \\
\hline $0047-579$ & $2007-11-10$ & 1.32 & 1.04 & 1.43 & 2.27 & 0.68 & 6.3 & SS & $4.2 \mathrm{E}+11$ & $2.68 \mathrm{E}+28$ & $3.08 \mathrm{E}+28$ \\
\hline $0208-512$ & 2007-11-10 & 1.62 & 2.23 & 2.54 & 1.83 & 0.71 & 6.2 & SS & $6.2 \mathrm{E}+12$ & $1.12 \mathrm{E}+28$ & $1.26 \mathrm{E}+28$ \\
\hline $0332-403$ & 2008-02-07 & 0.30 & 0.43 & 0.46 & 4.79 & 3.62 & -64.6 & SS & $2.3 \mathrm{E}+10$ & $5.67 \mathrm{E}+27$ & $5.75 E+27$ \\
\hline $0405-385$ & 2008-02-07 & 2.26 & 1.29 & 1.35 & 3.98 & 2.68 & -69.1 & SS & $1.6 \mathrm{E}+11$ & $1.24 \mathrm{E}+28$ & $1.26 \mathrm{E}+28$ \\
\hline $0438-436$ & 2008-02-07 & 1.47 & 0.83 & 1.42 & 4.58 & 3.21 & -70.1 & SS & $7.0 \mathrm{E}+10$ & $6.02 \mathrm{E}+28$ & $9.59 \mathrm{E}+28$ \\
\hline $0454-463$ & 2007-11-10 & 4.20 & 2.52 & 3.65 & 2.12 & 0.59 & 10.0 & SS & $3.0 \mathrm{E}+12$ & $8.77 \mathrm{E}+27$ & $1.22 \mathrm{E}+28$ \\
\hline $0506-612$ & 2007-11-10 & 0.96 & 0.85 & 0.93 & 1.62 & 0.74 & 7.1 & SS & $1.8 \mathrm{E}+12$ & $5.52 \mathrm{E}+27$ & $5.79 \mathrm{E}+27$ \\
\hline $0518-458$ & 2007-11-10 & 1.26 & 0.50 & 0.87 & 2.24 & 0.74 & 4.9 & SS & $2.1 E+11$ & $1.56 \mathrm{E}+24$ & $2.28 \mathrm{E}+24$ \\
\hline $0521-365$ & 2007-11-10 & 1.50 & 1.07 & 1.62 & 2.54 & 0.69 & 2.6 & SS & $1.2 \mathrm{E}+11$ & $4.04 \mathrm{E}+24$ & $1.09 \mathrm{E}+25$ \\
\hline $0537-441$ & 2007-11-10 & 3.30 & 4.89 & 5.13 & 2.17 & 0.70 & 11.6 & SS & $1.1 \mathrm{E}+14$ & $1.84 \mathrm{E}+28$ & $1.93 E+28$ \\
\hline $0625-354$ & 2007-11-10 & 0.30 & 0.29 & 0.36 & 2.91 & 0.90 & 4.7 & SS & $8.1 E+10$ & $1.99 \mathrm{E}+24$ & $2.35 \mathrm{E}+24$ \\
\hline $0637-752$ & 2008-02-07 & 1.86 & 2.67 & 3.47 & 3.95 & 0.93 & 66.5 & SS & $1.9 \mathrm{E}+11$ & $5.30 \mathrm{E}+27$ & $6.00 \mathrm{E}+27$ \\
\hline $1104-445$ & 2007-11-10 & 2.10 & 1.06 & 1.42 & 3.09 & 0.79 & 1.6 & SS & $8.4 \mathrm{E}+11$ & $1.88 \mathrm{E}+28$ & $2.28 \mathrm{E}+28$ \\
\hline $1144-379$ & 2008-02-07 & 1.30 & 1.68 & 1.77 & 5.14 & 3.24 & -75.1 & SS & $8.3 \mathrm{E}+11$ & $9.50 \mathrm{E}+27$ & $9.92 \mathrm{E}+27$ \\
\hline $1257-326$ & 2008-02-07 & 0.27 & 0.13 & 0.18 & 3.70 & 0.78 & -0.5 & SS & $8.1 E+10$ & $1.21 \mathrm{E}+27$ & $1.59 \mathrm{E}+27$ \\
\hline $1313-333$ & 2008-02-07 & 0.57 & 0.68 & 0.77 & 3.21 & 0.59 & -0.2 & SS & $3.6 \mathrm{E}+12 * *$ & $5.49 \mathrm{E}+27$ & $6.18 \mathrm{E}+27$ \\
\hline $1322-428$ & 2007-11-10 & 1.20 & 0.60 & 2.75 & 1.64 & 0.41 & 7.9 & DS & $3.7 \mathrm{E}+10$ & $8.03 E+21$ & $2.29 \mathrm{E}+22$ \\
\hline $1323-526$ & 2007-11-10 & 0.81 & 1.00 & 1.08 & 2.16 & 0.83 & 8.4 & SS & $1.3 \mathrm{E}+12 *$ & & \\
\hline $1333-337$ & 2008-02-07 & 0.36 & 0.17 & 0.24 & 3.74 & 0.97 & -7.4 & DS & $4.8 E+10$ & 6.071 & $7.67 \mathrm{E}+22$ \\
\hline $1424-418$ & 2007-11-10 & 0.84 & 1.15 & 1.58 & 2.38 & 0.68 & 3.4 & SS & $3.0 \mathrm{E}+12$ & 1.931 & $2.25 \mathrm{E}+28$ \\
\hline $1454-354$ & 2007-11-10 & 0.27 & 0.37 & 0.44 & 2.87 & 0.75 & 3.6 & SS & $3.3 \mathrm{E}+11$ & $4.86 \mathrm{E}+27$ & $5.30 \mathrm{E}+27$ \\
\hline $1501-343$ & 2007-11-10 & 0.30 & 0.24 & 0.24 & 2.76 & 0.68 & 3.6 & $\mathrm{C}$ & $1.9 \mathrm{E}+11 * * *$ & & \\
\hline $1549-790$ & 2008-02-07 & 0.48 & 0.28 & 1.37 & 1.96 & 0.62 & -0.3 & DS & $1.5 \mathrm{E}+10$ & $2.67 \mathrm{E}+25$ & $9.76 E+25$ \\
\hline $1610-771$ & 2008-02-07 & 2.19 & 0.86 & 1.83 & 1.40 & 0.50 & 5.2 & SS & $3.5 \mathrm{E}+11$ & $2.44 \mathrm{E}+28$ & $3.48 \mathrm{E}+28$ \\
\hline $1714-336$ & 2007-11-10 & 0.51 & 0.61 & 1.01 & 4.46 & 3.46 & -72.6 & SS & $1.8 \mathrm{E}+09^{*}$ & & \\
\hline $1716-771$ & 2007-11-10 & 0.57 & 0.12 & 0.14 & 1.33 & 0.83 & 13.5 & SS & $6.6 \mathrm{E}+10$ & $5.39 \mathrm{E}+22$ & $6.02 \mathrm{E}+22$ \\
\hline $1718-649$ & 2008-02-07 & 3.90 & 1.07 & 3.36 & 2.13 & 0.45 & -12.4 & Irr & $1.1 \mathrm{E}+11^{*}$ & & \\
\hline $1733-565$ & 2008-02-07 & 0.18 & 0.15 & 0.18 & 2.63 & 0.79 & -4.0 & DS & $4.5 \mathrm{E}+10$ & $3.75 E+24$ & $4.04 \mathrm{E}+24$ \\
\hline $1759-396$ & 2007-11-10 & 1.00 & 1.27 & 1.42 & 3.00 & 0.67 & 5.7 & SS & $2.4 \mathrm{E}+12$ & $3.35 \mathrm{E}+26$ & $3.70 \mathrm{E}+26$ \\
\hline $1804-502$ & 2007-11-10 & 0.39 & 0.32 & 0.33 & 2.60 & 0.67 & 8.6 & SS & $2.4 \mathrm{E}+12 * *$ & $5.23 \mathrm{E}+27$ & $5.37 \mathrm{E}+27$ \\
\hline $1814-637$ & 2008-02-07 & 1.70 & 0.37 & 0.93 & 1.92 & 0.61 & 2.3 & DS & $1.7 \mathrm{E}+11$ & $3.73 \mathrm{E}+24$ & $8.12 \mathrm{E}+24$ \\
\hline $1933-400$ & 2007-11-10 & 1.50 & 1.15 & 1.30 & 3.30 & 0.90 & 23.3 & SS & $2.0 \mathrm{E}+12$ & $5.32 \mathrm{E}+27$ & $5.93 \mathrm{E}+27$ \\
\hline $1954-388$ & 2008-02-07 & 1.92 & 1.28 & 2.30 & 3.09 & 0.38 & 5.0 & SS & $1.5 \mathrm{E}+12$ & $1.66 \mathrm{E}+27$ & $3.64 \mathrm{E}+27$ \\
\hline $2005-489$ & 2007-11-10 & 0.72 & 0.44 & 0.65 & 3.66 & 1.09 & 36.6 & SS & $2.5 \mathrm{E}+10$ & $6.13 \mathrm{E}+24$ & $7.36 \mathrm{E}+24$ \\
\hline 2027-308 & 2008-06-09 & 0.75 & 0.09 & 0.12 & 3.43 & 1.39 & -6.9 & SS & $4.1 \mathrm{E}+10^{* * *}$ & & \\
\hline $2052-474$ & 2008-02-07 & 0.60 & 1.49 & 1.67 & 3.19 & 0.47 & 12.8 & SS & $2.0 \mathrm{E}+12$ & $2.21 \mathrm{E}+28$ & $2.25 E+28$ \\
\hline $2106-413$ & 2008-02-07 & 1.50 & 0.78 & 1.30 & 2.51 & 0.46 & 10.4 & SS & $1.8 \mathrm{E}+11$ & $6.69 E+27$ & $7.46 \mathrm{E}+27$ \\
\hline 2149-306 & 2008-03-28 & 2.51 & 0.90 & 1.34 & 3.31 & 0.60 & -0.9 & SS & $9.3 \mathrm{E}+11$ & $4.06 \mathrm{E}+28$ & $5.56 \mathrm{E}+28$ \\
\hline $2152-699$ & 2008-02-07 & 0.33 & 0.38 & 0.51 & 3.38 & 0.75 & -4.0 & SS & $4.7 \mathrm{E}+10$ & $7.59 \mathrm{E}+23$ & $8.60 \mathrm{E}+23$ \\
\hline 2155-304 & 2008-03-28 & 0.60 & 0.39 & 0.51 & 3.98 & 0.62 & -1.5 & SS & $3.3 \mathrm{E}+10$ & $1.49 \mathrm{E}+25$ & $1.64 \mathrm{E}+25$ \\
\hline $2204-540$ & 2008-02-07 & 1.95 & 0.84 & 1.09 & 2.12 & 0.51 & -2.4 & SS & $6.0 \mathrm{E}+11$ & $7.09 \mathrm{E}+27$ & $8.67 \mathrm{E}+27$ \\
\hline $2326-477$ & 2007-11-10 & 2.52 & 0.52 & 1.06 & 2.01 & 0.60 & 6.8 & SS & $3.4 \mathrm{E}+11$ & $5.61 \mathrm{E}+27$ & $1.02 \mathrm{E}+28$ \\
\hline $2355-534$ & 2008-02-07 & 0.84 & 1.44 & 1.68 & 4.08 & 0.88 & -11.3 & SS & $1.2 \mathrm{E}+12$ & $7.50 \mathrm{E}+27$ & $8.50 \mathrm{E}+27$ \\
\hline
\end{tabular}

Notes. ${ }^{(a)}$ Usually $3 \times$ RMS noise in image. ${ }^{(b)}$ “*” indicates that $z=0$ was used as a limit. ${ }^{(c) ~ “ * * ” ~ i n d i c a t e s ~ t h a t ~ t h e ~ v a l u e ~ h a s ~ b e e n ~ c a l c u l a t e d ~ f r o m ~}$ theoretical SNR limits as described in Sect. 6.4. These limits were checked using all possible combinations of major axis and axial ratio using the difwrap package (Lovell 1998) and were found to be consistent.

multifrequency observation is needed in order to address this quantitatively. Thus 1814-637 appears to have a CSO morphology. Given the identification with a galaxy it is likely that this is a relatively young radio source. This source was considered as a possible EGRET detection by Edwards (2005).

1933-400 Based on VLA observations, Perley (1982) found that this source has a diffuse secondary component extending from the core to $3.5 \mathrm{arcsec}$ at a position angle of $140^{\circ}$. PKS 1933-400 is identified with 3EG J1935-4022 (Hartman et al. 1999; Edwards 2005) but the source was not in the initial Fermi 3-month bright source list. Our image shows a very linear jet to the southeast, emanating from a bright core.
1954-388 This source has a high optical polarization, up to $11 \%$ (Impey \& Tapia 1988, 1990) and a GPS-type spectrum (Tornikoski et al. 2001; Edwards \& Tingay 2004). VLBI observations showed a compact core (Preston et al. 1985; Shen et al. 1998b). Based on VLBA observations, Fomalont et al. (2000) present a elongation of the core to the southsouthwest, whereas Ojha et al. (2004a) found a weak component about 3 mas to the west of the core. Our TANAMI image shows a westward directed jet, extending about 5 mas from the core.

2005-489 This is one of the brightest known BL Lac sources (Wall et al. 1986) and it is classified as a high-frequency peaked BL Lac (HBL) due to its X-ray-to-radio flux ratio (Sambruna et al. 1995). It is a TeV source discovered by HESS, which 
Table 5. Source structure of tapered images.

\begin{tabular}{ccccccccc}
\hline \hline Source & $\begin{array}{c}\text { Epoch } \\
\text { yyyy-mm-dd }\end{array}$ & $\begin{array}{c}\text { Contour } \\
\left(\mathrm{mJy} \mathrm{beam}^{-1}\right)\end{array}$ & $\begin{array}{c}S_{\text {peak }} \\
\left(\mathrm{Jy} \mathrm{beam}^{-1}\right)\end{array}$ & $\begin{array}{c}S_{\text {total }} \\
(\mathrm{Jy})\end{array}$ & $\begin{array}{c}\theta_{\text {maj }} \\
(\mathrm{mas})\end{array}$ & $\begin{array}{c}\theta_{\text {min }} \\
(\mathrm{mas})\end{array}$ & $\begin{array}{c}\text { PA } \\
\left({ }^{\circ}\right)\end{array}$ & Taper $^{a}$ \\
\hline $0047-579$ & $2007-11-10$ & 1.25 & 1.25 & 1.42 & 4.32 & 3.71 & 86.2 & 100 \\
$0208-512$ & $2007-11-10$ & 1.15 & 2.32 & 2.51 & 4.32 & 3.52 & -82.8 & 100 \\
$0454-463$ & $2007-11-10$ & 2.49 & 3.11 & 3.52 & 2.78 & 1.22 & 11.2 & 100 \\
$0506-612$ & $2007-11-10$ & 0.90 & 0.90 & 0.97 & 5.61 & 4.70 & -83.8 & 50 \\
$0625-35$ & $2007-11-10$ & 3.00 & 0.32 & 0.36 & 5.59 & 4.59 & -71.0 & 50 \\
$0637-752$ & $2008-02-07$ & 1.50 & 3.12 & 3.51 & 6.55 & 5.24 & -47.3 & 50 \\
$1144-379$ & $2008-02-07$ & 0.55 & 1.69 & 1.77 & 7.33 & 4.45 & -71.8 & 50 \\
$1424-418$ & $2007-11-10$ & 1.37 & 1.37 & 1.53 & 4.79 & 3.64 & -60.0 & 75 \\
$1454-354$ & $2007-11-10$ & 0.30 & 0.40 & 0.43 & 4.89 & 3.69 & -60.4 & 100 \\
$1549-790$ & $2008-02-07$ & 0.63 & 0.27 & 1.35 & 5.09 & 3.67 & -79.5 & 100 \\
$1610-771$ & $2008-02-07$ & 4.20 & 1.40 & 1.79 & 5.31 & 4.14 & 65.0 & 100 \\
$1814-637$ & $2008-02-07$ & 1.70 & 0.48 & 0.93 & 7.16 & 6.24 & 62.4 & 35 \\
$2005-489$ & $2007-11-10$ & 0.53 & 0.53 & 0.64 & 7.56 & 5.09 & 84.6 & 35 \\
\hline
\end{tabular}

Notes. ${ }^{(a)}$ Baseline length in $M \lambda$ at which the visibility data were downweighted to $10 \%$.

has the softest VHE spectrum $(\Gamma=4.0)$ ever measured from a BL Lac (Aharonian et al. 2005a). The source was detected with EGRET (Lin et al. 1999) and is one of the bright $\gamma$-ray sources detected by Fermi in its first three months of operations. The VLBI image by Shen et al. (1998b) shows a compact core. The image by Ojha et al. (2005) reveals an additional component about 3 mas to the southwest of the core. Both our images in Figs. 6 and 11 show a low surface brightness jet to the southwest of the core, which seems to have a very wide opening angle.

2027-308 There is only very limited information on this source in the literature. Grandi (1983) note that it is probably a member of the class of very-narrow-line emission galaxies. Sowards-Emmerd et al. (2004) listed this source as a likely counterpart of the EGRET source 3EG 2034-3110. Our TANAMI image shows an unresolved core with a jet-like extension to the southwest.

2052-474 This source was identified with the EGRET source 3EG J2055-4716 (Hartman et al. 1999). Observations with Chandra did not reveal extended X-ray emission, but there is a two-sided arcsecond-scale radio jet in addition to the bright radio core (Marshall et al. 2005). The VLBI image by Ojha et al. (2004a) shows a compact core. Our image shows a very weak jet to the west.

2106-413 The radio core of this quasar is moderately polarized (3.5\%, Impey \& Tapia 1990). ATCA observations indicate that the radio spectrum peaks near $5 \mathrm{GHz}$ (Kollgaard et al. 1995). The VLBA image by Fomalont et al. (2000) shows a slightly elongated core. Our image shows a bright component about 2-3 mas east of the core, which appears elongated in the north-south direction and additional diffuse jet emission further to the east.

2149-306 This is a high-redshift, high-luminosity radio-loud quasar with a strongly blueshifted $\mathrm{Fe} \mathrm{K} \alpha$ line at $\sim 17 \mathrm{keV}$ in the quasar frame (Yaqoob et al. 1999; Wang et al. 2003). The VLBI map at $8.4 \mathrm{GHz}$ by Ojha et al. (2005) shows a component to the west of the core with a separation of 8.7 mas. This component is not seen in our TANAMI image, which rather shows jet emission within $\sim 5$ mas east of the core.

2152-699 This FR II radio source has a classic double-lobed structure (Fosbury et al. 1990), and is one of the brightest sources in the sky at $2.3 \mathrm{GHz}$ (Wall 1994). Tadhunter et al. (1988) find that the radio axis and optical emission line features on the kiloparsec-scale are misaligned and suggest interaction between the radio jet and an extra-nuclear cloud of gas. The parsecscale radio jet aligns strongly with optical emission line features (Tingay et al. 1996b). The VSOP image by Tingay et al. (2002) shows a resolved core and highly linear, narrow jet approximately 6 mas to the northeast. Ojha et al. (2004a) find a similar morphology consisting of the core and jet component to the northeast with separation of a few mas. Our image is in agreement with these previous images of this source, revealing well-collimated but knotty jet emission on intermediate scales out to about 30 mas from the core.

2155-304 This is one of the brightest extragalactic X-ray sources in the sky and was detected with most high-energy satellites including EGRET (Vestrand et al. 1995). TeV $\gamma$-rays from this source were observed (Aharonian et al. 2005b) and a TeV flare occured in July 2006 (Aharonian et al. 2007). The VLBI map presented by Ojha et al. (2004a) shows a compact core without additional components. Piner et al. (2008) could measure an apparent speed of a single jet component of $0.93 c \pm 0.31 c$ in a diffuse region $\sim 5$ mas southeast of the core. Our TANAMI image does show the same structure on the same scales but at somewhat higher dynamic range. In addition, there is a $\sim 15 \sigma$ component at the same position angle as the inner jet at $\sim 15$ mas from the core. The core itself has a relatively low brightness temperature of $T_{\mathrm{B}}=3.7 \times 10^{10} \mathrm{~K}$.

2204-540 This bright quasar is a member of our radio-selected subsample and has a high polarization of $6.6 \pm 0.2 \%$ (Ricci et al. 2004). There is only limited information on this source in the literature, which is surprising for such a bright object. It has not been seen by EGRET but it is among the brightest $\gamma$-ray sources seen by Fermi in its first three months of observations 
R. Ojha et al.: TANAMI first epoch $8.4 \mathrm{GHz}$ images
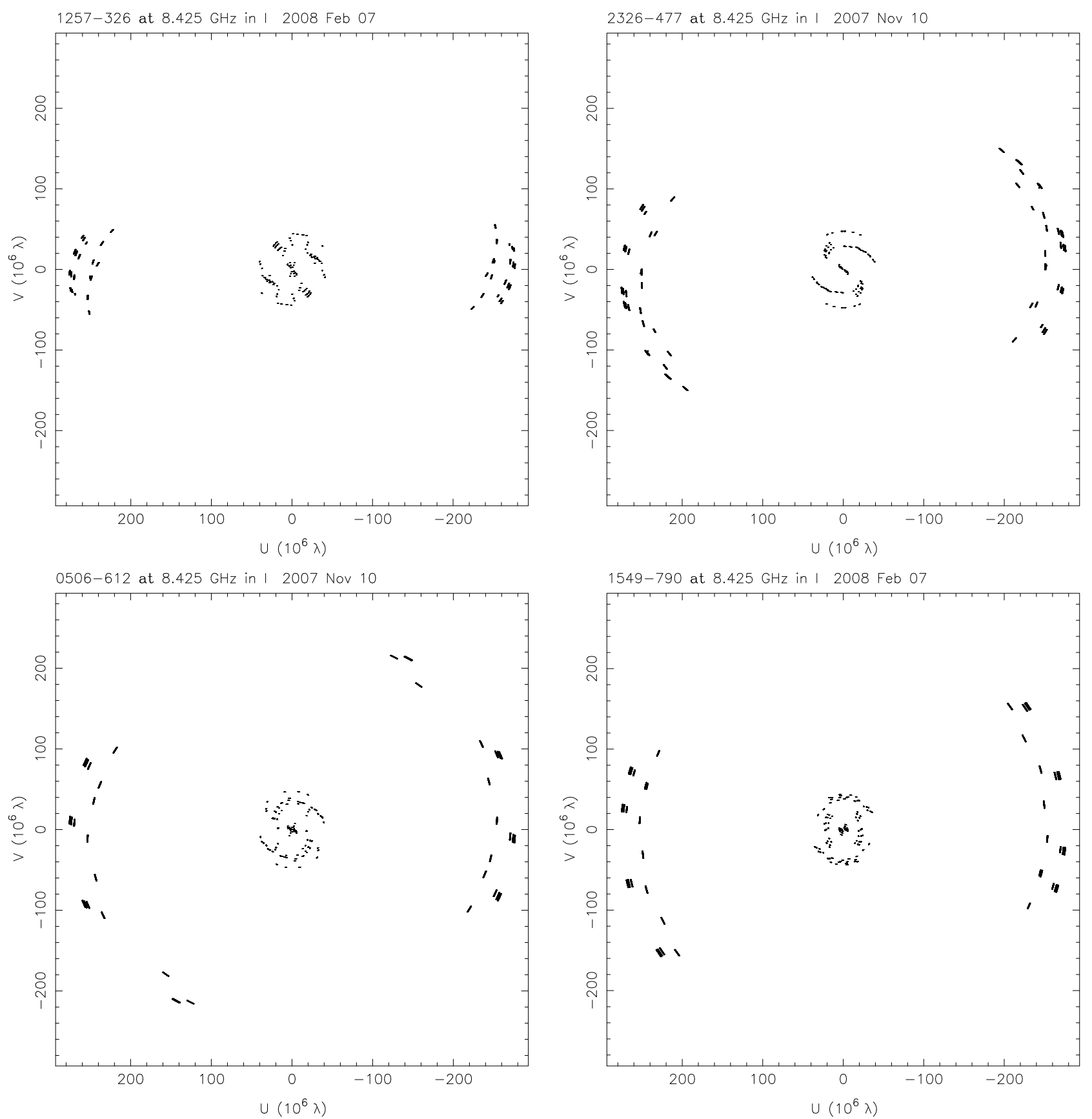

Fig. 12. The $(u, v)$-plane coverage of four sources chosen to span the declination range of the TANAMI targets and thus representative of them. The short baselines near the center of each plot are produced by the telescopes in Australia. The long baselines are furnished by the Hartebeesthoek telescope in South Africa. The "hole" in the (u,v)-plane coverage is a result of the absence of any telescopes between Australia and South Africa. Note that Hartebeesthoek is currently out of service and the long baselines are provided by O'Higgins in Antarctica and TIGO in Chile.

(Abdo et al. 2009b). Our image shows a bright high brightnesstemperature core and a short and possibly curved jet in the inner $\sim 4$ mas south and southwest of the core.

2326-477 Scott et al. (2004) find a compact, unresolved core structure smaller than 0.1 mas $^{2}$ of 410 mJy and a brightness temperature above $3 \times 10^{11} \mathrm{~K}$ at $5 \mathrm{GHz}$. Tingay et al. (2003b) find a substantially higher mean flux-density at $5 \mathrm{GHz}$ of $1.63 \mathrm{Jy}$ with ATCA and a moderate variability index of 0.05 , suggesting that a substantial fraction of the $5 \mathrm{GHz}$ total brightness is emitted on larger scales unresolved for the space-VLBI array. Our image shows jet-like emission peaking in two distinct components $\sim 6$ mas to the east and $\sim 10$ mas to the north-east of the compact core at $8.4 \mathrm{GHz}$.

2355-534 This is a optically violent and highly polarized source (Impey \& Tapia 1988, 1990). Shen et al. (1998b) present a VLBI image revealing a component to the southwest of the core with a separation of 4.9 mas. Our image shows at least two distinct jet components along the same position angle, an inner one at $\sim 3.5$ mas, which may consist of two subcomponents, and an outer one at $\sim 12$ mas. No $\gamma$-ray detection of this object has been reported so far. 


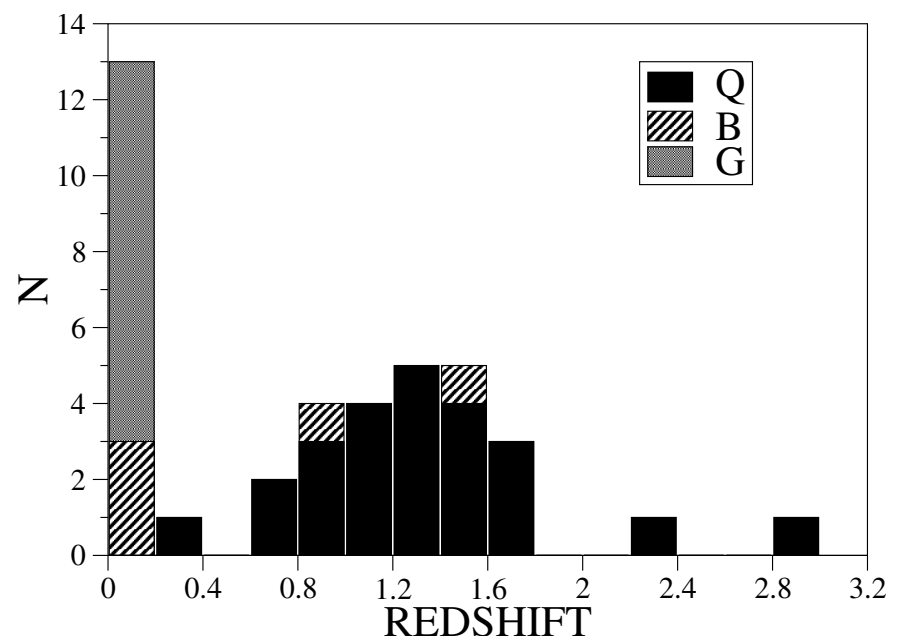

Fig. 13. Distribution of the redshifts of all TANAMI sources with optical identification shown.

\section{Discussion}

\subsection{Redshifts}

Figure 13 shows the redshift-distribution of all the TANAMI sources. For galaxies and BL Lac objects the distribution peaks at $z<0.4$ while for quasars it peaks at $\sim 1.5$, with a maximum redshift of 3. This is similar to the distribution observed for the LAT Bright AGN Sample (LBAS; Abdo et al. 2009b) and for the EGRET blazars (Mukherjee et al. 1997). The number of very low redshift AGN detected with the Fermi LAT is expected to increase as fainter sources become visible and more nearby radio galaxies are detected. The distributions of the radioselected and $\gamma$-ray selected sub-samples are shown separately in Figs. 14 and 15, respectively. There is no significant difference in the redshift distribution of these two sub-samples which is consistent with the strong link between bright AGN and $\gamma$-ray emission. Figure 16 again shows the overall redshift distribution of all TANAMI sources but this time with the LAT detections and non-detections indicated, based on the 3-month LAT bright-source list (Abdo et al. 2009b). A comparison of Figs. 15 and 16 shows that the redshift distribution of EGRET detected AGN is similar to that of LAT detected AGN. Figure 16 shows broadly similar redshift distributions for the LAT detected and non-detected AGN in the TANAMI sample with two interesting exceptions. First, most galaxies have not yet been detected by the LAT. Rather more curiously, none of the five most distant sources have been detected by the LAT. As the size of the TANAMI sample is increased, the interesting subsamples will become large enough to allow exhaustive statistical tests which can better quantify the above similarities and differences.

\subsection{Luminosities}

For all 38 TANAMI sources that have a published redshift, the core and the total luminosity was calculated assuming isotropic emission. The results are shown in the final two columns of Table 4. For both LAT detected and non-detected sources, the values range from about $10^{22}$ to almost $10^{29} \mathrm{~W} \mathrm{~Hz}^{-1}$ with two thirds of both of these categories of sources having total luminosity above $10^{27} \mathrm{~W} \mathrm{~Hz}^{-1}$. There is a clear difference between the distribution of luminosity of different optical types with all eight galaxy luminosities below $10^{26} \mathrm{~W} \mathrm{~Hz}^{-1}$ and all twentyfour quasar luminosities above $10^{26} \mathrm{~W} \mathrm{~Hz}^{-1}$ (all but one above

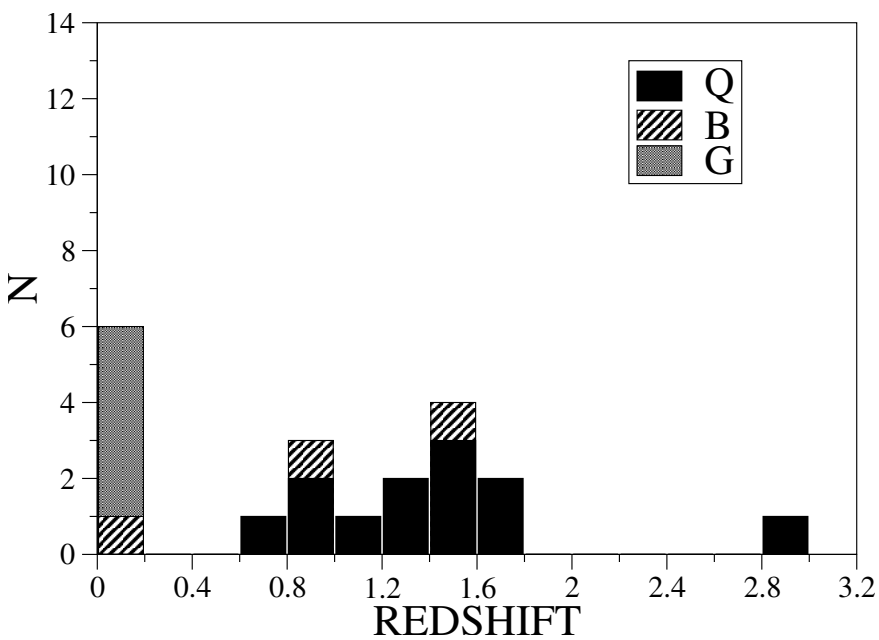

Fig. 14. Distribution of the redshifts of the radio-selected sub-sample of TANAMI sources.

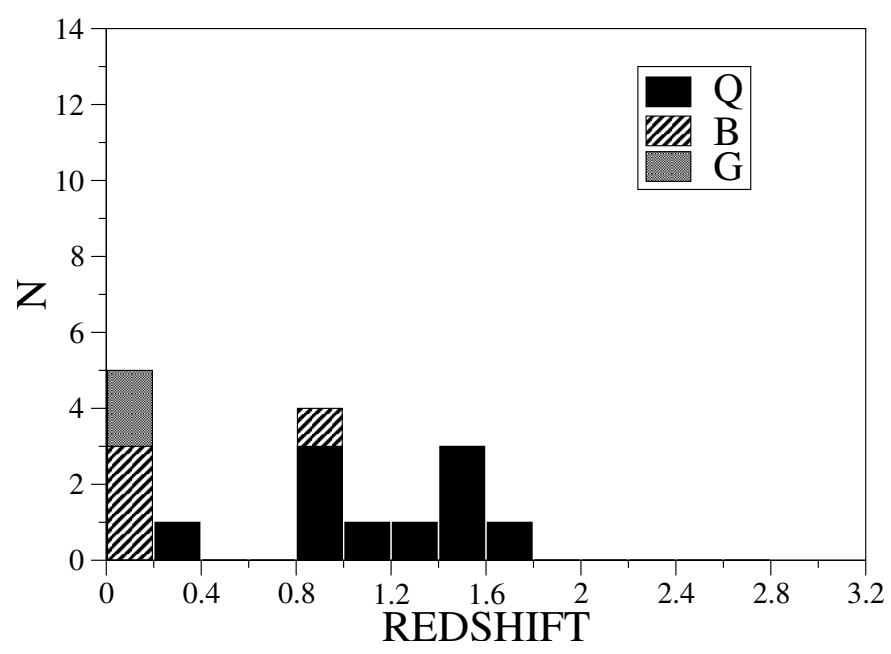

Fig. 15. Distribution of the redshifts of the EGRET-selected sub-sample of TANAMI sources.

$10^{27} \mathrm{~W} \mathrm{~Hz}^{-1}$ ). The five BLLacs in the sample are evenly distributed between $10^{24}$ and $10^{28} \mathrm{~W} \mathrm{~Hz}^{-1}$.

None of the five most luminous sources (which are also the five most distant sources, see 6.1) have been detected by the LAT. More intriguing, none of the nine most luminous jets (obtained by taking the difference of total and core luminosity) are detected which, taken at face value, would suggest an unexpected anticorrelation between jet luminosity and $\gamma$-ray brightness. More typically, the four most luminous BLLac sources have been detected by the LAT.

\subsection{Morphology and connection to Fermi}

To discuss the morphology of TANAMI sources we have adopted the classification scheme used by Kellermann et al. (1998) which places objects into four categories. Sources that appear barely resolved are considered "compact" (C), those with the most compact component at either end of the image are considered "single-sided" (SS) and those with the most compact component in the middle of the image are considered "doublesided" (DS). Finally, there is a category of sources with "irregular" (Irr) structure which includes sources with morphology that does not fall into the first three categories. This scheme has the 
R. Ojha et al.: TANAMI first epoch $8.4 \mathrm{GHz}$ images

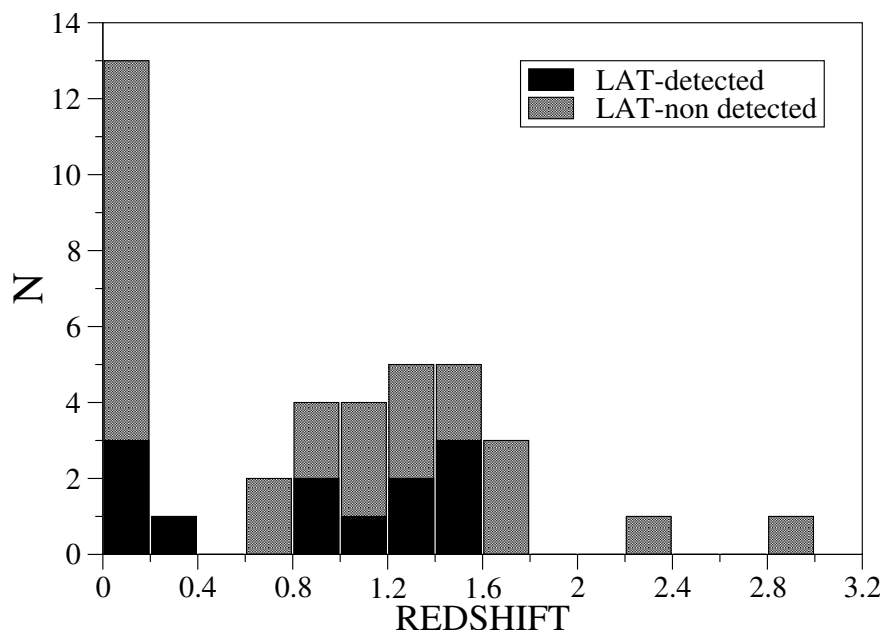

Fig. 16. Distribution of the redshifts of all TANAMI sources with LAT detections and non-detections shown. While broadly similar, most galaxies and none of the five most distant sources have been detected yet by the LAT.

virtue of not making any assumptions about the physical nature of the objects under study, separating the description of the observations from their interpretation. For an excellent discussion of the possible physical conditions associated with these categories see Sect. 4 of Kellermann et al. (1998).

Three TANAMI sources, 0332-403, 0405-385, and 1501-343, appear compact from our images. However, we only classify 1501-343 as "compact" since our images for the first two sources have a lower resolution due to the absence of trans-oceanic baselines and past images indicate the presence of some weak extended structure. Optically 1501-343 is an "unclassified" source. 1322-428, 1333-337, 1549-790, 1733-566 and 1814-637 are double-sided. All five are galaxies. Another galaxy, 1718-649 is the only "irregular" source in our sample. All of the remaining 36 sources ( $84 \%$ of the TANAMI sample) are single-sided (Table 6). These include all quasars, all BL Lacs, four of the ten galaxies and two of the three optically unidentified sources in the TANAMI sample. Characteristics of individual sources are discussed in Sect. 5.

Since the $\gamma$-ray emission is likely to be beamed and orientation dependent, we would expect to find differences in the parsec scale morphology of $\gamma$-loud and $\gamma$-quiet objects. However, past comparisons of VLBI morphologies of EGRET detected sources with those not detected by EGRET failed to show any connection between observed $\gamma$-ray emission and parsec scale structure (Taylor et al. 2007). This was attributed to the fact that nearly all EGRET sources were detected only when flaring and that most sources lay within a factor of 10 of EGRET's minimum detectable flux. The expectation is that essentially all bright compact radio sources will be $\gamma$-ray loud if observed with significantly better sensitivity than EGRET.

After three months of observations Fermi found 12 of our 43 TANAMI sources (28\%) to be bright $\gamma$-ray sources with $>10 \sigma$ significance. This includes, 11 of the $36 \mathrm{SS}$ sources. However, only one (Centaurus A) of the 5 DS sources have been detected so far. In terms of optical identification, 11 of the Fermi detections are quasars and BL Lacs with Cen A the sole galaxy detected. This is consistent with the canonical picture that the "core-jet" nature of quasars and BL Lacs is the result of differential Doppler boosting of an intrinsically symmetric twin-jet. That such AGN are associated with $\gamma$-ray emission was a key finding
Table 6. Distribution of morphology.

\begin{tabular}{lrr}
\hline \hline Structure $^{a}$ & Number & $\%$ \\
\hline SS & 36 & 84 \\
C & 1 & 2 \\
DS & 5 & 12 \\
Irr & 1 & 2 \\
\hline
\end{tabular}

Notes. ${ }^{(a)}$ Classification of the structure, as follows: (C) compact, (SS) single-sided, (DS) double-sided, or (Irr) irregular.

of EGRET and is consistent with theoretical models. None of the three optically unidentified sources in our sample have been detected so far.

Interestingly, two of the four known intraday variable (IDV) sources in our sample (0405-385 and 1144-379) have been detected by Fermi. All four IDV sources have a "SS" morphology. This is consistent with our current understanding of IDV sources as being among the most extreme members of the blazar class.

To compare the opening angles of Fermi-detected and nondetected blazar jets, we fitted circular Gaussian components to the visibility data and measured the angle at which the innermost jet component appears from the position of the core of the jet (Table 7). In two cases (0332-403 and 0405-385) the lack of long baselines did not allow us to resolve the inner jet structure and to model-fit any jet components. In one case (0454-463), the jet was too weak and partially resolved, and in one case (1501-343), the source was unresolved even on the longest baselines. We excluded all galaxies from this analysis, first, because we cannot unambiguously determine the core position in a number of galaxy jet-counterjet systems and, second, because galaxies are usually not detected by Fermi. Our initial analysis suggests that 7 out of $9(78 \%)$ LBAS sources have an opening angle $>30$ degrees while only 4 out of 15 (27\%) nonLBAS sources have an opening angle $>30$ degrees. This would suggest that $\gamma$-ray bright jets have either smaller Lorentz factors (the width of the relativistic beaming cone is $\sim 1 / \Gamma$ ) or that they are pointed closer to the line of sight than $\gamma$-ray faint jets. An inverse correlation between $\gamma$-ray brightness and the beaming seems very unlikely and, in fact, previous studies have shown that the Lorentz factor of $\gamma$-ray brighter jets as seen with Fermi are higher than for $\gamma$-ray fainter jets (e.g., Lister et al. 2009b; Kovalev et al. 2009). If confirmed by future analysis of larger samples, the result that $\gamma$-ray brighter jets have larger observed opening angles might thus imply that these appear geometrically increased in projection because of smaller angles to the line of sight (Pushkarev et al. 2009).

\subsection{Brightness temperature}

We identified the core component for each source based on its morphology and, in some cases, past VLBI images and identifications. In all cases, this approach led to the identification of the core as the brightest and most compact feature, either at the end of a one-sided jet or at the center of a double-sided twinjet. Gaussian models were fitted to the core visibility data using DIFMAP. We did this by replacing the CLEAN components from the core region in the final model with an elliptical Gaussian component. The brightness temperature of the fitted core component was then calculated in the rest frame of the source using the expression:

$T_{\mathrm{B}}=\frac{2 \ln 2}{\pi k} \frac{S_{\text {core }} \lambda^{2}(1+z)}{\theta_{\text {maj }} \theta_{\min }}$ 
Table 7. Opening angles of TANAMI sources.

\begin{tabular}{rrrrrrr}
\hline \hline Source & Class & $\begin{array}{r}r^{1} \\
\text { (mas) }\end{array}$ & $\begin{array}{r}\theta^{2} \\
\text { (degrees) }\end{array}$ & $\begin{array}{r}\text { Component size } \\
\text { (mas) }\end{array}$ & $\begin{array}{r}\text { Opening angle } \\
\text { (degrees) }\end{array}$ & Limit? $^{3}$ \\
\hline $0047-579$ & $\mathrm{Q}$ & 2.11 & -13.0 & 0.26 & 7.2 & $\mathrm{Y}$ \\
$0208-512$ & $\mathrm{~B}$ & 2.93 & 24.3 & 1.88 & 35.6 & $\mathrm{~N}$ \\
$0438-436$ & $\mathrm{Q}$ & 6.54 & -44.8 & 1.36 & 11.9 & $\mathrm{~N}$ \\
$0506-612$ & $\mathrm{Q}$ & 3.02 & -46.1 & 0.19 & 3.7 & $\mathrm{Y}$ \\
$0521-365$ & $\mathrm{~B}$ & 3.04 & -37.2 & 0.36 & 6.9 & $\mathrm{~N}$ \\
$0537-441$ & $\mathrm{Q}$ & 2.57 & 57.2 & 1.41 & 30.9 & $\mathrm{~N}$ \\
$0637-752$ & $\mathrm{Q}$ & 6.08 & 1.5 & 0.42 & 4.0 & $\mathrm{Y}$ \\
$1104-445$ & $\mathrm{Q}$ & 3.12 & 27.2 & 2.14 & 38.2 & $\mathrm{~N}$ \\
$1144-379$ & $\mathrm{Q}$ & 1.02 & -54.3 & 0.65 & 36.4 & $\mathrm{~N}$ \\
$1257-326$ & $\mathrm{Q}$ & 2.8 & -36.4 & 1.51 & 30.7 & $\mathrm{~N}$ \\
$1313-333$ & $\mathrm{Q}$ & 3.74 & -7.5 & 2.45 & 36.3 & $\mathrm{~N}$ \\
$1323-526$ & $\mathrm{U}$ & 3.26 & 83.2 & 0.41 & 7.3 & $\mathrm{~N}$ \\
$1424-418$ & $\mathrm{Q}$ & 0.98 & 42.5 & 0.87 & 47.9 & $\mathrm{~N}$ \\
$1454-354$ & $\mathrm{Q}$ & 1.77 & 2.6 & 0.87 & 27.5 & $\mathrm{~N}$ \\
$1610-771$ & $\mathrm{Q}$ & 2.16 & -48.1 & 0.40 & 10.7 & $\mathrm{~N}$ \\
$1714-336$ & $\mathrm{~B}$ & 13.61 & 32.7 & 4.73 & 19.7 & $\mathrm{~N}$ \\
$1716-771$ & $\mathrm{U}$ & 2.3 & 49.8 & 0.30 & 7.4 & $\mathrm{Y}$ \\
$1759-396$ & $\mathrm{Q}$ & 2.61 & -74.6 & 1.67 & 36.2 & $\mathrm{~N}$ \\
$1804-502$ & $\mathrm{Q}$ & 2.29 & 54.1 & 0.96 & 23.9 & $\mathrm{~N}$ \\
$1933-400$ & $\mathrm{Q}$ & 2.39 & -51.0 & 0.31 & 7.6 & $\mathrm{Y}$ \\
$1954-388$ & $\mathrm{Q}$ & 1.55 & -44.1 & 0.45 & 16.5 & $\mathrm{~N}$ \\
$2005-489$ & $\mathrm{~B}$ & 1.52 & 31.8 & 1.18 & 42.4 & $\mathrm{~N}$ \\
$2052-474$ & $\mathrm{Q}$ & 1.91 & -31.0 & 0.27 & 8.0 & $\mathrm{~N}$ \\
$2106-413$ & $\mathrm{Q}$ & 3.1 & 1.7 & 0.47 & 8.6 & $\mathrm{Y}$ \\
$2149-306$ & $\mathrm{Q}$ & 2.49 & -28.1 & 0.26 & 6.0 & $\mathrm{Y}$ \\
$2155-304$ & $\mathrm{~B}$ & 2.72 & -56.2 & 1.74 & 35.4 & $\mathrm{~N}$ \\
$2204-540$ & $\mathrm{Q}$ & 2.14 & 64.6 & 0.30 & 8.0 & $\mathrm{~N}$ \\
$2326-477$ & $\mathrm{Q}$ & 3.47 & -11.0 & 0.30 & 5.0 & $\mathrm{Y}$ \\
$2355-534$ & $\mathrm{Q}$ & 3.88 & 37.9 & 0.49 & 7.3 & $\mathrm{~N}$ \\
\hline
\end{tabular}

Notes. ${ }^{(1)}$ Radial distance between core and fitted component. ${ }^{(2)}$ Angle between core and fitted component. ${ }^{(3)}$ Flag indicating whether the innermost jet component is unresolved, resulting in an upper limit on the opening angle. $\mathrm{Y}=$ yes, $\mathrm{N}=$ no.

where $S_{\text {core }}$ is the flux density of the core and $\theta_{\text {maj }}$ and $\theta_{\text {min }}$ are the major and minor axis FWHMs, respectively, of the Gaussian component. $k$ is the Boltzmann constant, $z$ the redshift and $\lambda$ the wavelength of observation (compare Kovalev et al. 2005).

In order to distinguish between cores that were resolved and those that were not we compared the fitted component sizes $\theta_{\text {fit }}$ with the theoretical resolution limit of our array $\theta_{\text {lim }}$. We calculated $\theta_{\text {lim }}$ taking into account the synthesized beamsize, $\theta_{\text {beam }}$ (where $\theta_{\text {beam }}$ is the geometric mean of the major and minor beam axes) and the signal-to-noise ratio (SNR) of the core component following Kovalev et al. (2005, Eq. (2)). Components with fitted sizes $\theta_{\text {fit }}<\theta_{\text {lim }}$ are considered unresolved and we determine lower limits of their calculated brightness temperatures by using $\theta_{\lim }$ as an upper limit on their size. It should be noted that all other brightness temperatures are limits in a similar sense, because VLBI observations at a given frequency can never rule out the possibility that even smaller structures inside the cores are dominating the core emission.

The core brightness temperatures for all sources are listed in Table 4 and their distribution is shown in Fig. 17. The higher end of the distribution is dominated by quasars while the lowest brightness temperatures are those of BL Lacs and galaxies. We find 2 sources with fully unresolved cores (1501-343, and 2027-308) and 2 which are unresolved perpendicular to the jet axis and resolved along it (1313-333 and 1804-502). Fourteen sources show a maximum brightness temperature below the equipartition value of $10^{11} \mathrm{~K}$ (Readhead 1994) with thirty sources having values below the inverse Compton limit

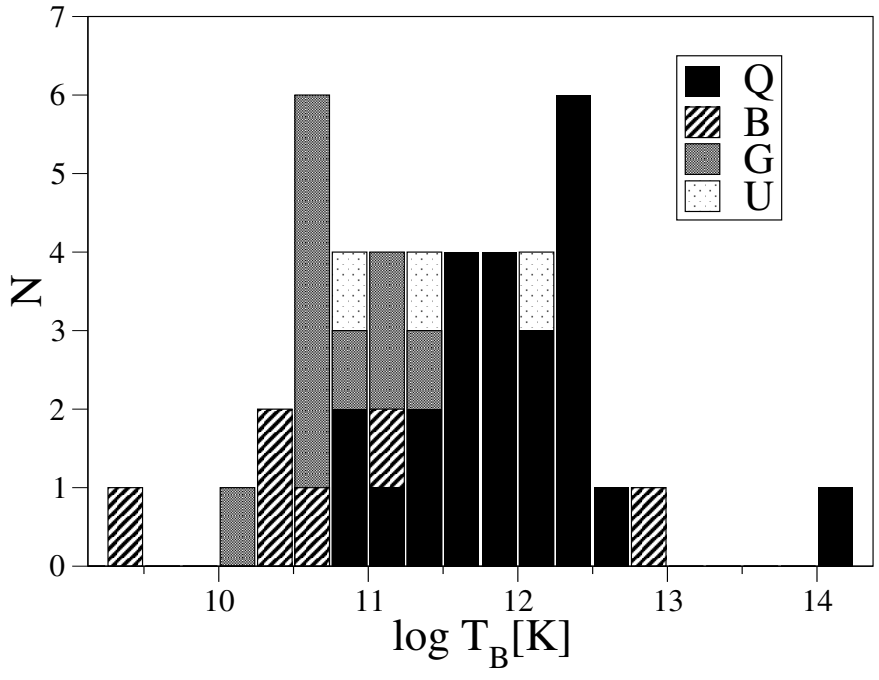

Fig. 17. Distribution of core brightness temperature of all TANAMI sources with optical identification shown.

of $10^{12} \mathrm{~K}$ described by Kellermann \& Pauliny-Toth (1969). As many as thirteen sources exceed this limit, one of them substantially (0537-441). This is most likely a result of Doppler boosting that is commonly seen in blazars. However, contributions from exotic mechanisms such as coherent emission and relativistic proton emission and/or non-simple geometries 


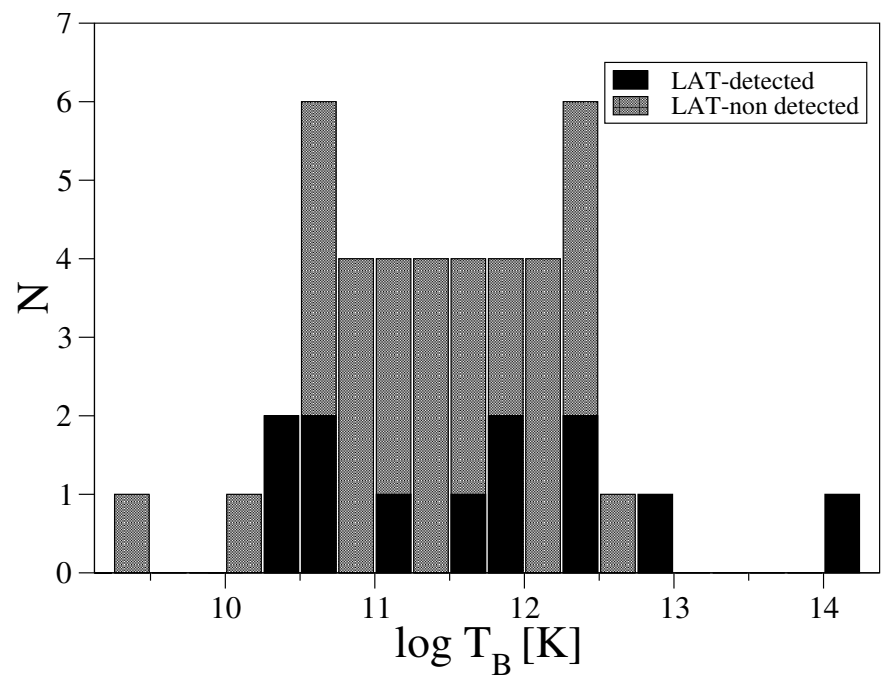

Fig. 18. Distribution of core brightness temperature of all TANAMI sources with LAT detections and non-detections shown.

cannot be ruled out (Kellermann et al. 2004). The median value is near $2.1 \times 10^{11} \mathrm{~K}$ with the maximum value exceeding $10^{14} \mathrm{~K}$. This is comparable to the results of the MOJAVE sample (Kovalev et al. 2005).

Figure 18 shows the core brightness temperatures of LAT detections and non-detections, based on the 3-month LAT list. There does not appear to be any significant difference between LAT detections and non-detections. It is interesting to note that many sources with high brightness temperatures, and thus expected to have high Doppler factors, remain undetected by the LAT. Nine of the thirteen with values above $10^{12} \mathrm{~K}$ remain undetected.

\section{Conclusions}

Our first epoch $8.4 \mathrm{GHz}$ TANAMI images show that the Australian Long Baseline Array and associated telescopes provide high quality images of $\gamma$-ray blazars that can be used to study the physics of blazars. In many cases these images represent a substantial improvement on published work. The addition of telescopes in Antarctica and Chile is expected to compensate for the loss of the Hartebeesthoek telescope in South Africa and to improve the current $(u, v)$-coverage.

The TANAMI sample has been defined as a hybrid radio and $\gamma$-ray selected sample of AGN south of $\delta=-30^{\circ}$. Of this sample, $84 \%$ of sources show a one-sided morphology, $12 \%$ (all galaxies) are double sided, while one optically unidentified source is compact and one galaxy has a irregular morphology. Of these quasars and BL Lacs have similar morphologies, all being single-sided. The ten galaxies in the sample include five double sided objects, one irregular object and four single sided objects.

About $28 \%$ of TANAMI sources have been detected by the Fermi LAT after three months of observations. When galaxies are excluded, initial analysis shows that $78 \%$ of sources detected by the LAT have opening angles $>30$ degrees compared to just $27 \%$ of non-LBAS sources. This suggests that $\gamma$-ray bright jets are pointed closer to the line of sight than $\gamma$-ray faint jets, with the observed opening angles appearing geometrically increased. This result should be regarded as preliminary owing to the modest number of sources available for analysis at this time.

The redshift distribution of the BL Lacs and quasars in the TANAMI sample is similar to the distributions seen for the
LBAS and EGRET blazars. No difference is seen between the radio- and $\gamma$-ray selected subsamples. The redshift distributions of EGRET and LAT detected AGN are also similar. However, most galaxies and none of the five most distant and most luminous sources have been detected by the LAT. Galaxies have the lowest luminosities, BLLacs are more luminous (than galaxies) as a group while quasars dominate the high end of the luminosity distribution. None of the nine most luminous jets have been detected by the LAT so far.

The high end of the brightness temperature distribution is dominated by quasars with the lower end composed mostly of BL Lacs and galaxies. Fourteen sources have a maximum brightness temperature below the equipartition value, and 30 sources have a value below the inverse Compton limit putting about a third of the values above this limit. The median value is near $3 \times 10^{11} \mathrm{~K}$ with the maximum value exceeding $10^{14} \mathrm{~K}$. There does not appear to be any significant difference in the brightness temperature distributions of LAT detections and non-detections. Many of the sources with very high brightness temperatures have not yet been detected by the LAT.

The initial results presented here will be augmented by $22 \mathrm{GHz}$ images as well as multi-epoch data in future papers to address our scientific questions including those that require spectral and kinematic information. Of particular, interest will be TANAMI epochs observed since the launch of Fermi satellite thus providing near-simultaneous data at radio, $\gamma$-ray as well as at intermediate wavelengths from coordinated observations with other instruments. As discussed in Sect. 1, TANAMI and Fermi data will also be used with neutrino data from ANTARES and KM3NET for the identification and study of neutrino point sources.

Acknowledgements. We are grateful to Dirk Behrend, Neil Gehrels, Julie McEnery, David Murphy, and John Reynolds, who contributed in numerous ways to the success of the TANAMI program so far. Furthermore, we thank the Fermi/LAT AGN group for the good collaboration. The Long Baseline Array is part of the Australia Telescope which is funded by the Commonwealth of Australia for operation as a National Facility managed by CSIRO. This work made use of the Swinburne University of Technology software correlator, developed as part of the Australian Major National Research Facilities Programme and operated under licence. M.K. has been supported in part by an appointment to the NASA Postdoctoral Program at the Goddard Space Flight Center, administered by Oak Ridge Associated Universities through a contract with NASA. We would like to thank the staff of the Swinburne correlator for their unflagging support. This research has made use of data from the NASA/IPAC Extragalactic Database (NED, operated by the Jet Propulsion Laboratory, California Institute of Technology, under contract with the National Aeronautics and Space Administration); and the SIMBAD database (operated at CDS, Strasbourg, France). This research has made use of NASA's Astrophysics Data System. This research has made use of the United States Naval Observatory (USNO) Radio Reference Frame Image Database (RRFID).

\section{References}

Abdo, A. A., Ackermann, M., Ajello, M., et al. 2009a, ApJS, 183, 46 Abdo, A. A., Ackermann, M., Ajello, M., et al. 2009b, ApJ, 700, 597 Abdo, A. A., Ackermann, M., Atwood, W. B., et al. 2009c, ApJ, 697, 934 Aharonian, F., Akhperjanian, A. G., Aye, K. M., et al. 2005a, A\&A, 436, L17 Aharonian, F., Akhperjanian, A. G., Aye, K. M., et al. 2005b, A\&A, 430, 865 Aharonian, F., Akhperjanian, A. G., Bazer-Bachi, A. R., et al. 2007, ApJ, 664, L71

Atwood, W. B., Abdo, A. A., Ackermann, M., et al. 2009, ApJ, 697, 1071 Bignall, H. E., Jauncey, D. L., Lovell, J. E. J., et al. 2003, ApJ, 585, 653 Bignall, H. E., Macquart, J. P., Jauncey, D. L., et al. 2006, ApJ, 652, 1050 Bignall, H., Cimo, G., Jauncey, D., et al. 2008, Proceedings of Science in From

Planets to Dark Energy: the Modern Radio Universe PoS (MRU), 084 Boisson, C., Cayatte, V., \& Sol, H. 1989, A\&A, 211, 275

Böttcher, M. 2007, Ap\&SS, 309, 95

Browne, I. W. A., Savage, A., \& Bolton, J. G. 1975, MNRAS, 173, 87P 
Bryant, J. J., \& Hunstead, R. W. 2002, MNRAS, 337, 861

Cassaro, P., Stanghellini, C., Bondi, M., et al. 1999, A\&AS, 139, 601 Chartas, G., Worrall, D. M., Birkinshaw, M., et al. 2000, ApJ, 542, 655 Chiang, J., Fichtel, C. E., von Montigny, C., et al. 1995, ApJ, 452, 156 Christiansen, W. N., Frater, R. H., Watkinson, A., et al. 1977, MNRAS, 181, 183 Cohen, M. H., Lister, M. L., Homan, D. C., et al. 2007, ApJ, 658, 232 da Costa, L. N., Pellegrini, P. S., Davis, M., et al. 1991, ApJS, 75, 935 Danziger, I. J., \& Goss, W. M. 1979, MNRAS, 186, 93

Danziger, I. J., Fosbury, R. A. E., Goss, W. M., \& Ekers, R. D. 1979, MNRAS, 188,415

Deller, A. T., Tingay, S. J., Bailes, M., \& West, C. 2007, PASP, 119, 318

Dodson, R., Fomalont, E. B., Wiik, K., et al. 2008, ApJS, 175, 314

Dondi, L., \& Ghisellini, G. 1995, MNRAS, 273, 583

Drinkwater, M. J., Webster, R. L., Francis, P. J., et al. 1997, MNRAS, 284, 85

Edwards, P. G. 2005, Ap\&SS, 297, 31

Edwards, P. G., \& Tingay, S. J. 2004, A\&A, 424, 91

Edwards, P. G., Piner, B. G., Tingay, S. J., et al. 2006, PASJ, 58, 233

Falomo, R., Maraschi, L., Treves, A., \& Tanzi, E. G. 1987, ApJ, 318, L39

Falomo, R., Pesce, J. E., \& Treves, A. 1993, ApJ, 411, L63

Fanaroff, B. L., \& Riley, J. M. 1974, MNRAS, 167, 31P

Fey, A. L., Clegg, A. W., \& Fomalont, E. B. 1996, ApJS, 105, 299

Fomalont, E. B., Frey, S., Paragi, Z., et al. 2000, ApJS, 131, 95

Fosbury, R. A. E., di Serego Alighieri, S., Courvoisier, T. J. L., et al. 1990, in ed. E. Rolfe, ESA Spec. Publ., 310, 513

Gehrels, N., \& Michelson, P. 1999, Astrop. Phys., 11, 277

Goss, W. M., Wellington, K. J., Christiansen, W. N., et al. 1977, MNRAS, 178, 525

Govoni, F., Falomo, R., Fasano, G., \& Scarpa, R. 2000, A\&AS, 143, 369

Graham, J. A. 1978, PASP, 90, 237

Grandi, S. A. 1983, MNRAS, 204, 691

Greisen, E. W. 1998, in Astronomical Data Analysis Software and Systems VII, ed. R. Albrecht, R. N. Hook, \& H. A.Bushouse, ASP Conf. Ser., 145, 204 Hartman, R. C., Bertsch, D. L., Fichtel, C. E., et al. 1992, ApJ, 385, L1

Hartman, R. C., Bertsch, D. L., Bloom, S. D., et al. 1999, ApJS, 123, 79 Hewitt, A., \& Burbidge, G. 1989, A new optical catalog of QSO Holt, J., Tadhunter, C., Morganti, R., et al. 2006, MNRAS, 370, 1633 Horiuchi, S., Fomalont, E. B., Taylor, W. K., et al. 2004, ApJ, 616, 110 Horiuchi, S., Meier, D. L., Preston, R. A., \& Tingay, S. J. 2006, PASJ, 58, 211 Hunstead, R. W., \& Murdoch, H. S. 1980, MNRAS, 192, 31P

Hunstead, R. W., Murdoch, H. S., \& Shobbrook, R. R. 1978, MNRAS, 185, 149 Hunstead, R. W., Durdin, J. M., Little, A. G., et al. 1982, Proc. Astron. Soc. Austr., 4, 447

Impey, C. D., \& Tapia, S. 1988, ApJ, 333, 666

Impey, C. D., \& Tapia, S. 1990, ApJ, 354, 124

Jackson, C. A., Wall, J. V., Shaver, P. A., et al. 2002, A\&A, 386, 97

Jauncey, D. L., Batty, M. J., Gulkis, S., \& Savage, A. 1982, AJ, 87, 763

Jauncey, D. L., Batty, M. J., Wright, A. E., et al. 1984, ApJ, 286, 498

Kadler, M., Ros, E., Lobanov, A. P., et al. 2004, A\&A, 426, 481

Kedziora-Chudczer, L. 2006, MNRAS, 369, 449

Keel, W. C. 1985, AJ, 90, 2207

Kellermann, K. I., \& Pauliny-Toth, I. I. K. 1969, ApJ, 155, L71

Kellermann, K. I., Vermeulen, R. C., Zensus, J. A., \& Cohen, M. H. 1998, AJ, 115,1295

Kellermann, K. I., Lister, M. L., Homan, D. C., et al. 2004, ApJ, 609, 539

Killeen, N. E. B., Bicknell, G. V., \& Ekers, R. D. 1986, ApJ, 302, 306

Kollgaard, R. I., Feigelson, E. D., Laurent-Muehleisen, S. A., et al. 1995, ApJ, 449, 61

Kovalev, Y. Y., Kellermann, K. I., Lister, M. L., et al. 2005, AJ, 130, 2473

Kovalev, Y. Y., Aller, H. D., Aller, M. F., et al. 2009, ApJ, 696, L17

Kuehr, H., Witzel, A., Pauliny-Toth, I. I. K., \& Nauber, U. 1981, A\&AS, 45, 367

Lauberts, A., \& Valentijn, E. A., 1989, The Messenger, 56, 31

Liang, E. W., \& Liu, H. T. 2003, MNRAS, 340, 632

Lin, Y. C., Bertsch, D. L., Bloom, S. D., et al. 1997, in Proceedings of the Fourth Compton Symposium, ed. C. D. Dermer, M. S. Strickman, \& J. D. Kurfess, Am. Inst. Phys. Conf. Ser., 410, 1371

Lin, Y. C., Bertsch, D. L., Bloom, S. D., et al. 1999, ApJ, 525, 191

Lister, M. L., Aller, H. D., Aller, M. F., et al. 2009a, AJ, 137, 3718

Lister, M. L., Homan, D. C., Kadler, M., et al. 2009b, ApJ, 696, L22

Marelli, M. 2008, The Astronomer's Telegram, 1701, 1

Marshall, H. L., Schwartz, D. A., Lovell, J. E. J., et al. 2005, ApJS, 156, 13

Mattox, J. R., Hartman, R. C., \& Reimer, O. 2001, ApJS, 135, 155

McCulloch, P. M., Ellingsen, S. P., Jauncey, D. L., et al. 2005, AJ, 129, 2034

Morganti, R., Oosterloo, T. A., Tadhunter, C. N., et al. 2001, MNRAS, 323, 331

Morton, D. C., Savage, A., \& Bolton, J. G. 1978, MNRAS, 185, 735

Mukherjee, R., Bertsch, D. L., Bloom, S. D., et al. 1997, ApJ, 490, 116

Nicolson, G. D., Glass, I. S., Feast, M. W., \& Andrews, P. J. 1979, MNRAS, 189, 29P

Nolan, P. L., Bertsch, D. L., Chiang, J., et al. 1996, ApJ, 459, 100
Ojha, R., Fey, A. L., Johnston, K. J., et al. 2004a, AJ, 127, 3609 Ojha, R., Fey, A. L., Johnston, K. J., et al. 2004b, AJ, 127, 1977 Ojha, R., Fey, A. L., Charlot, P., et al. 2005, AJ, 130, 2529

Pellegrini, S., Venturi, T., Comastri, A., et al. 2003, ApJ, 585, 677 Perley, R. A. 1982, AJ, 87, 859

Perlman, E. S., Padovani, P., Giommi, P., et al. 1998, AJ, 115, 1253

Peterson, B. A., Jauncey, D. L., Condon, J. J., \& Wright, A. E. 1976, ApJ, 207, L5

Petrov, L., Hirota, T., Honma, M., et al. 2007, AJ, 133, 2487

Pian, E., Falomo, R., Hartman, R. C., et al. 2002, A\&A, 392, 407

Piner, B. G., Pant, N., \& Edwards, P. G. 2008, ApJ, 678, 64

Preston, R. A., Morabito, D. D., Williams, J. G., et al. 1985, AJ, 90, 1599

Preston, R. A., Jauncey, D. L., Meier, D. L., et al. 1989, AJ, 98, 1

Pushkarev, A. B., Kovalev, Y. Y., Lister, M. L., \& Savolainen, T. 2009, A\&A, 507, L33

Quintana, H., \& Ramirez, A. 1995, ApJS, 96, 343

Readhead, A. C. S. 1994, ApJ, 426, 51

Ricci, R., Prandoni, I., Gruppioni, C., et al. 2004, A\&A, 415, 549

Sambruna, R. M., Urry, C. M., Ghisellini, G., \& Maraschi, L. 1995, ApJ, 449, 567

Scarpa, R., Falomo, R., \& Pian, E. 1995, A\&A, 303, 730

Scarpa, R., Urry, C. M., Falomo, R., \& Treves, A. 1999, ApJ, 526, 643

Schwartz, D. A., Marshall, H. L., Lovell, J. E. J., et al. 2000, ApJ, 540, 69

Scott, W. K., Fomalont, E. B., Horiuchi, S., et al. 2004, ApJS, 155, 33

Shen, Z. Q., Wan, T. S., Moran, J. M., et al. 1997, AJ, 114, 1999

Shen, Z. Q., Hong, X. Y., \& Wan, T. S. 1998a, Chinese Astronomy and Astrophysics, 22, 133

Shen, Z. Q., Wan, T. S., Moran, J. M., et al. 1998b, AJ, 115, 1357

Shepherd, M. C. 1997, in Astronomical Data Analysis Software and Systems VI, ed. G. Hunt, \& H. Payne, ASP Conf. Ser., 125, 77

Smith, R. J., Lucey, J. R., Hudson, M. J., et al. 2000, MNRAS, 313, 469

Sowards-Emmerd, D., Romani, R. W., Michelson, P. F., \& Ulvestad, J. S. 2004, ApJ, 609, 564

Stickel, M., Fried, J. W., \& Kuehr, H. 1989, A\&AS, 80, 103

Stickel, M., Meisenheimer, K., \& Kuehr, H. 1994, A\&AS, 105, 211

Sulentic, J. W., Stirpe, G. M., Marziani, P., et al. 2004, A\&A, 423, 121

Tadhunter, C. N., Fosbury, R. A. E., di Serego Alighieri, S., et al. 1988, MNRAS, 235,403

Tadhunter, C. N., Morganti, R., di Serego-Alighieri, S., et al. 1993, MNRAS, 263, 999

Tadhunter, C., Wills, K., Morganti, R., et al. 2001, MNRAS, 327, 227

Taylor, G. B., Healey, S. E., Helmboldt, J. F., et al. 2007, ApJ, 671, 1355

Thompson, D. J., Bertsch, D. L., Dingus, B. L., et al. 1993a, ApJ, 415, L13

Thompson, D. J., Bertsch, D. L., Fichtel, C. E., et al. 1993b, ApJ, 410, 87

Tingay, S. J., \& Edwards, P. G. 2002, AJ, 124, 652

Tingay, S. J., \& de Kool, M. 2003, AJ, 126, 723

Tingay, S. J., Edwards, P. G., Costa, M. E., et al. 1996a, ApJ, 464, 170

Tingay, S. J., Jauncey, D. L., Reynolds, J. E., et al. 1996b, AJ, 111, 718

Tingay, S. J., Jauncey, D. L., Reynolds, J. E., et al. 1997, AJ, 113, 2025

Tingay, S. J., Jauncey, D. L., Reynolds, J. E., et al. 2000, AJ, 119, 1695

Tingay, S. J., Reynolds, J. E., Tzioumis, A. K., et al. 2002, ApJS, 141, 311

Tingay, S. J., Edwards, P. G., \& Tzioumis, A. K. 2003a, MNRAS, 346, 327

Tingay, S. J., Jauncey, D. L., King, E. A., et al. 2003b, PASJ, 55, 351

Tornikoski, M., Jussila, I., Johansson, P., et al. 2001, AJ, 121, 1306

Tornikoski, M., Lähteenmäki, A., Lainela, M., \& Valtaoja, E. 2002, ApJ, 579, 136

Tosti, G. 2008, The Astronomer's Telegram, 1759, 1

Tzioumis, A., King, E., Morganti, R., et al. 2002, A\&A, 392, 841

Ulrich, M. H. 1981, A\&A, 103, L1

Venturi, T., Morganti, R., Tzioumis, T., \& Reynolds, J. 2000, A\&A, 363, 84 Veron-Cetty, M. P., \& Veron, P. 2006, VizieR Online Data Catalog, 7248, 0 Vestrand, W. T., Stacy, J. G., \& Sreekumar, P. 1995, ApJ, 454, L93 von Montigny, C., Bertsch, D. L., Chiang, J., et al. 1995, ApJ, 440, 525 Wall, J. V. 1994, Austr. J. Phys., 47, 625

Wall, J. V., Danziger, I. J., Pettini, M., et al. 1986, in Quasars, ed. G. Swarup, \& V. K. Kapahi, IAU Symp., 119, 59

Wang, J., Yaqoob, T., Szokoly, G., et al. 2003, ApJ, 590, L87

Waxman, E. 2007, Science, 315, 63

White, G. L., Jauncey, D. L., Wright, A. E., et al. 1988, ApJ, 327, 561

Wills, B. J., Wills, D., Breger, M., et al. 1992, ApJ, 398, 454

Wills, K. A., Morganti, R., Tadhunter, C. N., et al. 2004, MNRAS, 347, 771

Wilson, A. S., Young, A. J., \& Shopbell, P. L. 2001, ApJ, 547, 740

Wisotzki, L., Christlieb, N., Bade, N., et al. 2000, A\&A, 358, 77

Yaqoob, T., George, I. M., Turner, T. J., et al. 1998, ApJ, 505, L87

Yaqoob, T., George, I. M., Nandra, K., et al. 1999, ApJ, 525, L9

Zensus, J. A., Ros, E., Kellermann, K. I., et al. 2002, AJ, 124, 662 
1 United States Naval Observatory, 3450 Massachusetts Ave., NW, Washington DC 20392, USA

e-mail: rojha@usno.navy.mil

2 NVI, Inc., 7257D Hanover Parkway, Greenbelt, MD 20770, USA

3 Dr. Remeis Sternwarte, Astron. Institut der Universität ErlangenNürnberg, Sternwartstrasse 7, 96049 Bamberg, Germany

e-mail: matthias.kadler@sternwarte.uni-erlangen.de

${ }^{4}$ Erlangen Centre for Astroparticle Physics, Erwin-Rommel Str. 1, 91058 Erlangen, Germany

5 CRESST/NASA Goddard Space Flight Center, Greenbelt, MD 20771, USA

6 Universities Space Research Association, 10211 Wincopin Circle, Suite 500 Columbia, MD 21044, USA

7 Hartebeesthoek Radio Astronomy Observatory, PO Box 443, Krugersdorp 1740, South Africa

8 The Catholic University of America, 620 Michigan Ave., N.E., Washington, DC 20064, USA

9 Australia Telescope National Facility, CSIRO, PO Box 76, Epping, NSW 1710, Australia

10 Max-Planck-Institut für Radioastronomie, Auf dem Hügel 69, 53121 Bonn, Germany

11 Bundesamt fur Kartographie und Geodesie, Germany entrusted with Transportable Integrated Geodetic Observatory, Universidad de Concepcion, Casilla 4036, Correo 3, Concepcion, Chile
12 Canberra Deep Space Communication Complex, PO Box 1035, Tuggeranong, ACT 2901, Australia

13 Department of Physics, Purdue University, 525 Northwestern Avenue, West Lafayette, IN 47907, USA

14 School of Mathematics \& Physics, Private Bag 37, University of Tasmania, Hobart TAS 7001, Australia

15 Federal Agency for Cartography and Geodesy (BKG), Geodetic Observatory Wettzell, Sackenrieder Str. 25, 93444 Bad Kötzting, Germany

16 Dept. d'Astronomia i Astrofísica, Universitat de València, 46100 Burjassot, València, Spain

17 Department of Physics and Astronomy, University of New Mexico, Albuquerque NM, 87131, USA $^{8}$

18 Astrophysics Science Division, NASA Goddard Space Flight Center, Greenbelt, MD 20771, USA

19 Curtin Institute of Radio Astronomy, Curtin University of Technology, Bentley, WA, 6102, Australia

20 Istituto Nazionale di Fisica Nucleare, Sezione di Perugia, 06123 Perugia, Italy

21 Dipartimento di Fisica, Università degli Studi di Perugia, 06123 Perugia, Italy

\footnotetext{
${ }^{8}$ Greg Taylor is also an Adjunct Astronomer at the National Radio Astronomy Observatory.
} 